\title{
A RECURSIVE FORMULA FOR THE JONES POLYNOMIAL OF 2-BRIDGE LINKS AND APPLICATIONS
}

\author{
Eunju Lee, Sang Youl Lee, And Myoungsoo Seo
}

\begin{abstract}
In this paper, we give a recursive formula for the Jones polynomial of a 2-bridge knot or link with Conway normal form $C\left(-2 n_{1}\right.$, $\left.2 n_{2},-2 n_{3}, \ldots,(-1)^{r} 2 n_{r}\right)$ in terms of $n_{1}, n_{2}, \ldots, n_{r}$. As applications, we also give a recursive formula for the Jones polynomial of a 3-periodic link $L^{(3)}$ with rational quotient $L=C\left(2, n_{1},-2, n_{2}, \ldots, n_{r},(-1)^{r} 2\right)$ for any nonzero integers $n_{1}, n_{2}, \ldots, n_{r}$ and give a formula for the span of the Jones polynomial of $L^{(3)}$ in terms of $n_{1}, n_{2}, \ldots, n_{r}$ with $n_{i} \neq \pm 1$ for all $i=1,2, \ldots, r$
\end{abstract}

\section{Introduction}

The Jones polynomial of an oriented link in $S^{3}$ was first introduced in [4]. Kauffman [8] and Murasugi [15] have used the Jones polynomial in verifying Tait conjecture which states that a reduced alternating diagram has minimal crossing number. Let $D$ be a connected, prime diagram of an oriented link $L$. Then the span of the Jones polynomial of $L$ is less than or equal to the number of crossings of $D$ and the equality holds if and only if $D$ is reduced alternating $[8,15,23]$.

In 1956, a characterization of 2-bridge knots and links was introduced by Schubert [21]. In [1], Conway introduced another presentation, now called Conway normal form, of 2-bridge knots and links. Several people have studied the Jones polynomials of 2-bridge knots and links [5, 6, 13, 14, 18, 19, 22]. In 1987, Lichorish and Millett [13] gave an algorithm to calculate the Homfly polynomials of 2-bridge knots and links with matrix manipulations. In 2002, Nakabo [19] also presented an explicit formula of the Homfly polynomials of 2bridge knots and links. Lu and Zhong [14] computed the Kauffman polynomials of 2-bridge knots and links using the Kauffman skein theory and linear algebra techniques. Note that the Jones polynomial can be obtained from the Homfly and Kauffman polynomials by substituting variables.

Received November 8, 2007.

2000 Mathematics Subject Classification. Primary 57M25.

Key words and phrases. Jones polynomial, 2-bridge knot, span, periodic link with rational quotient.

This work was supported for two years by Pusan National University Research Grant.

(C)2009 The Korean Mathematical Society 
On the other hand, Hilden, Lozano, and Montesinos-Amilibia [2] introduced a special kind of Conway normal form of a 2-bridge link with two components and studied the excellent component of the character variety of periodic knots in $S^{3}$ with rational quotients. In [16], Murasugi described several relationships between the Jones polynomials of a periodic link and its factor link. It is remarkable that the set of periodic links with rational quotients is a special family of periodic links which contains all 2-bridge knots and links, all torus knots and links and some pretzel knots and links, etc. It is known that every 2-bridge knot or link is a 2-periodic link with rational quotient and every 2periodic link with rational quotient is a 2-bridge knot or link [3]. The second and third authors [10] re-examined Hilden, Lozano, and Montesinos-Amilibia's presentation to study the Alexander polynomials of 2-bridge links with Conway normal form $C\left(2, n_{1},-2, n_{2}, \ldots, n_{r},(-1)^{r} 2\right)$ and $q$-periodic links in $S^{3}$ with rational quotients $C\left(2, n_{1},-2, n_{2}, \ldots, n_{r},(-1)^{r} 2\right)$ in terms of $n_{1}, n_{2}, \ldots, n_{r}$ and its period $q$. Thereafter, some properties for the family of periodic links with rational quotients are studied $[3,9,10,11,12]$.

In this paper, we first give a recursive formula for the Jones polynomial of a 2-periodic link with rational quotient, which is actually a recursive formula for the Jones polynomial of a 2-bridge knot or link. Generalizing this formula, we also obtain a recursive formula for the Jones polynomial of a 3-periodic link with rational quotient and a formula for the span of the Jones polynomial of this kind of 3-periodic link.

This paper is organized as follows. In Section 2, we review presentations of 2-bridge knots and links and periodic links with rational quotients. Section 3 contains the definition of bracket polynomial and formulas for periodic links with rational quotients. In Section 4, for arbitrary given nonzero integers $n_{1}, n_{2}, \ldots, n_{r}$, we give a recursive formula for the Jones polynomial of a 2-bridge knot or link with Conway normal form $C\left(-2 n_{1}, 2 n_{2},-2 n_{3}\right.$, $\left.\ldots,(-1)^{r} 2 n_{r}\right)$ in terms of $n_{1}, n_{2}, \ldots, n_{r}$. In Section 5 , we give a recursive formula for the Jones polynomial of a 3-periodic link $L^{(3)}$ with rational quotient $L=C\left(2, n_{1},-2, n_{2}, \ldots, n_{r},(-1)^{r} 2\right)$ for arbitrary given nonzero integers $n_{1}, n_{2}, \ldots, n_{r}$ and give a formula for the span of the Jones polynomial of $L^{(3)}$ in terms of $n_{1}, n_{2}, \ldots, n_{r}$ with $n_{i} \neq \pm 1$ for all $i=1,2, \ldots, r$. The formula for the span gives a lower bound for the minimal crossing number of the 3 -periodic link $L^{(3)}$.

\section{Periodic links with rational quotients}

To each pair $(\alpha, \beta)$ of two co-prime integers subject to the condition that $\beta$ is odd and $0<|\beta|<\alpha$, Schubert [21] associated an oriented diagram on the 2-sphere $S^{2}$ of an oriented 2-bridge $\operatorname{knot}\left(\alpha\right.$ odd) or $\operatorname{link}\left(\alpha\right.$ even) $L$ in $S^{3}$, now called the Schubert normal form of $L$ and denoted by $S(\alpha, \beta)$, and showed that any (oriented) 2-bridge knots and links in $S^{3}$ can be represented in this way. Two such pairs of integers $(\alpha, \beta)$ and $\left(\alpha^{\prime}, \beta^{\prime}\right)$ define an equivalent oriented 
(resp. unoriented) knot or link if and only if

$$
\alpha=\alpha^{\prime} \text { and } \beta^{ \pm 1} \equiv \beta^{\prime} \bmod 2 \alpha(\operatorname{resp} . \bmod \alpha),
$$

where $\beta^{-1}$ denotes the integer with the properties $0<\beta^{-1}<2 \alpha$ and $\beta \beta^{-1} \equiv$ $1 \bmod 2 \alpha$.

Let $\left[a_{1}, a_{2}, \ldots, a_{n}\right]$ denote a continued fraction expansion of $\alpha / \beta$ :

$$
\left[a_{1}, a_{2}, \ldots, a_{n}\right] \equiv a_{1}+\frac{1}{a_{2}+\frac{1}{\ddots \cdot+\frac{1}{a_{n}}}}=\frac{\alpha}{\beta} .
$$

Then $L=S(\alpha, \beta)$ has also a diagram $C\left(a_{1}, a_{2}, \ldots, a_{n}\right)$, called Conway normal form of $L$, as shown in Figure 1, depending on whether $n$ is even or odd [1]. The integral tangles in Figure 1, which are rectangles labeled $a_{i}$, are the 2-braids with $\left|a_{i}\right|$ crossings as shown in Figure 2. It is well known that $L=S(\alpha, \beta)$ admits a diagram $C\left(2 b_{1}, 2 b_{2}, \ldots, 2 b_{m}\right)$, which is equivalent to $C\left(a_{1}, a_{2}, \ldots, a_{n}\right)$ [7].
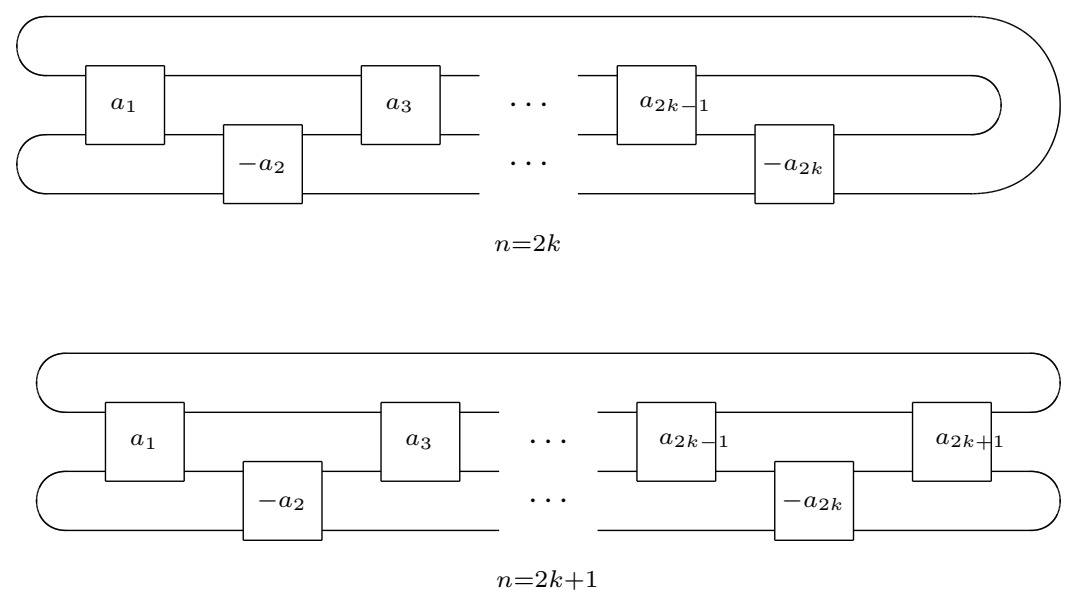

Figure 1

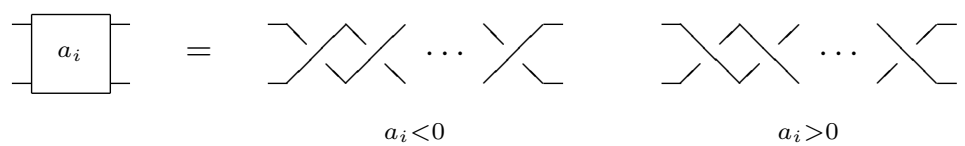

Figure 2

It is known $[2,10]$ that the 2-bridge link $L=S(\alpha, \beta)$ ( $\alpha$ even) can also be represented by Conway diagram of the form $C\left(2, n_{1},-2, n_{2}, \ldots, n_{r},(-1)^{r} 2\right)$ as 
shown in Figure 3. We choose an orientation of the 2-bridge link $C\left(2, n_{1},-2, n_{2}\right.$, $\left.\ldots, n_{r},(-1)^{r} 2\right)$ as shown in Figure 3 . Then it is easy to see that the diagram shown in Figure 3 can be deformed to the diagrams in Figure 4 by using Reidemeister moves. Throughout this paper, an oriented 2-bridge link $L$ in $S^{3}$ represented by the Conway normal form $C\left(2, n_{1},-2, n_{2}, \ldots, n_{r},(-1)^{r} 2\right)$ is denoted by $L=\vec{C}\left[\left[n_{1}, n_{2}, \ldots, n_{r}\right]\right]$.

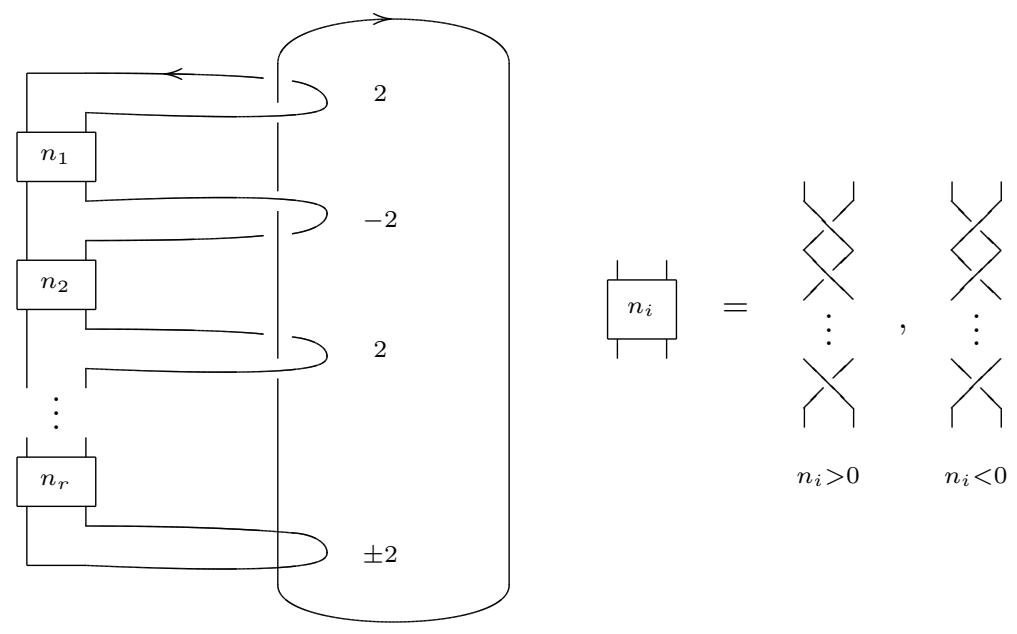

FiguRe 3
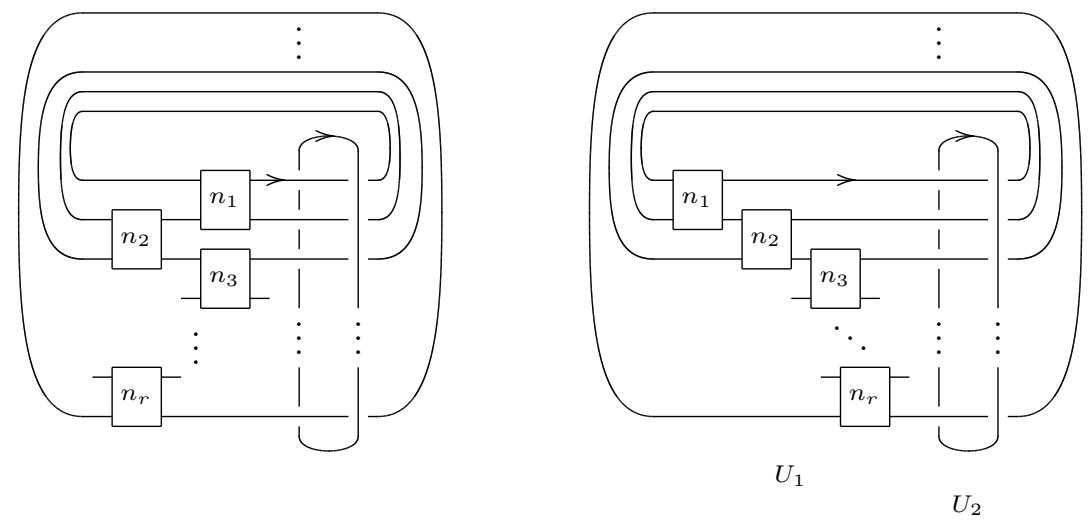

FIGURE 4 
A link $L$ in $S^{3}$ is called a $p$-periodic link ( $p \geq 2$ an integer) if there exists an orientation preserving auto-homeomorphism $h$ of $S^{3}$ such that $h(L)=L, h$ is of order $p$ and the set $\operatorname{Fix}(h)$ of fixed points of $h$ is a circle disjoint from $L$. In this case, the link $L /\langle h\rangle \cup \operatorname{Fix}(h)$ in the orbit space $S^{3} /\langle h\rangle \cong S^{3}$ is called the quotient link of $L$. Let $K$ be an oriented link in $S^{3}$ and $U$ an oriented trivial knot with $K \cap U=\emptyset$. For any integer $p \geq 2$, let $\phi_{U}^{p}: \Sigma^{3} \rightarrow S^{3}$ be a $p$-fold branched cyclic covering branched along $U$. Then $\Sigma^{3}$ is homeomorphic to the 3 -sphere $S^{3}$, and $\left(\phi_{U}^{p}\right)^{-1}(K)$ is a $p$-periodic link in $\Sigma^{3}$ with $L=K \cup U$ as its quotient link. We give an orientation to $\left(\phi_{U}^{p}\right)^{-1}(K)$ induced by the orientation of $K$. Note that any periodic knot or link in $S^{3}$ arises in this manner.

Definition ([10]). A link $\tilde{L}$ in $S^{3}$ is called a p-periodic link with rational quotient if it is a $p$-periodic link whose quotient link is a 2-bridge link, or equivalently, if there exists a 2-bridge link $L=U_{1} \cup U_{2}$ in $S^{3}$ such that $\tilde{L}$ is equivalent to the preimage $\left(\phi_{U_{2}}^{p}\right)^{-1}\left(U_{1}\right)$ of the component $U_{1}$ of $L$ by a $p$-fold cyclic covering $\phi_{U_{2}}^{p}: \Sigma^{3} \rightarrow S^{3}$ branched along the component $U_{2}$ of $L$.

Note that each component $U_{1}$ and $U_{2}$ of $L$ is a trivial knot and they can be interchanged each other by an orientation preserving homeomorphism of $S^{3}$ [17]. This implies that $\left(\phi_{U_{2}}^{p}\right)^{-1}\left(U_{1}\right)$ is equivalent to $\left(\phi_{U_{1}}^{p}\right)^{-1}\left(U_{2}\right)$. Now let $L=$ $\vec{C}\left[\left[n_{1}, n_{2}, \ldots, n_{r}\right]\right]=U_{1} \cup U_{2}$ be an oriented 2-bridge link as shown Figure 4. Then the diagram $D^{(p)}$ shown in Figure 5 is a canonical oriented $p$-periodic diagram of the oriented $p$-periodic link $\left(\phi_{U_{2}}^{p}\right)^{-1}\left(U_{1}\right)$ with rational quotient $L=$ $\vec{C}\left[\left[n_{1}, n_{2}, \ldots, n_{r}\right]\right]$. In what follows, we shall denote the oriented $p$-periodic link $\left(\phi_{U_{2}}^{p}\right)^{-1}\left(U_{1}\right)$ by $L^{(p)}$ or $\vec{C}\left[\left[n_{1}, n_{2}, \ldots, n_{r}\right]\right]^{(p)}$ for our convenience. Then any $p$ periodic link with rational quotient can be represented by $\vec{C}\left[\left[n_{1}, n_{2}, \ldots, n_{r}\right]\right]^{(p)}$ for some nonzero integers $n_{1}, n_{2}, \ldots, n_{r}[3,10]$.

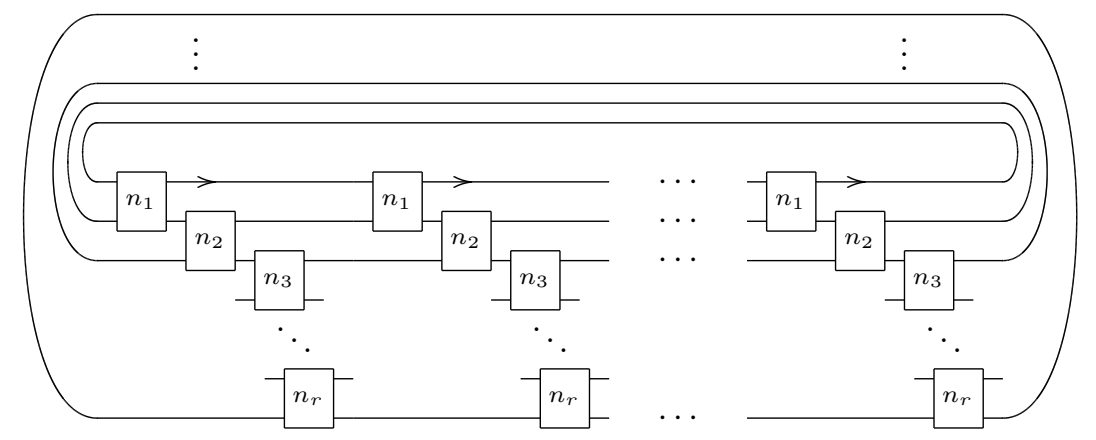

Figure 5. The canonical $p$-periodic diagram $D^{(p)}$ of $L^{(p)}$ 


\section{Bracket polynomial of periodic links}

The bracket polynomial of an unoriented link diagram $D$, denoted by $\langle D\rangle$, is a Laurent polynomial in a single variable $A$ defined by the following three axioms:

1. If $\bigcirc$ denotes the standard diagram of the unknot, then

$$
\langle\bigcirc\rangle=1
$$

2. If $\delta=-A^{-2}-A^{2}$ and $D \sqcup \bigcirc$ denotes the diagram $D$ together with the standard diagram of the unknot, disjoint from $D$, then

$$
\langle D \sqcup \bigcirc\rangle=\delta\langle D\rangle \text {. }
$$

3. Suppose that $D_{+}, D_{0}$ and $D_{\infty}$ are the diagrams that are exactly the same except at a neighborhood of one crossing point in which the diagrams differ as shown in Figure 6. Then

$$
\left\langle D_{+}\right\rangle=A\left\langle D_{\infty}\right\rangle+A^{-1}\left\langle D_{0}\right\rangle .
$$

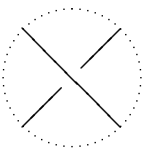

$D_{+}$

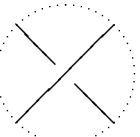

$D_{-}$

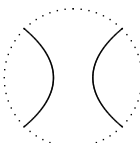

$D_{0}$

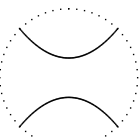

$D_{\infty}$

FIGURE 6

From (3), we also obtain the equation

$$
\left\langle D_{-}\right\rangle=A\left\langle D_{0}\right\rangle+A^{-1}\left\langle D_{\infty}\right\rangle .
$$

It is easy to see that $\langle D\rangle$ is an invariant under Reidemeister moves II and III, but not an invariant under Reidemeister move I. If $\varphi_{+}, \varphi_{-}$and $\varphi_{0}$ are the diagrams that are exactly the same except at a neighborhood of one crossing point in which the diagrams differ as shown in Figure 7, then we have

$$
\left\langle\varphi_{+}\right\rangle=(-A)^{3}\left\langle\varphi_{0}\right\rangle,\left\langle\varphi_{-}\right\rangle=(-A)^{-3}\left\langle\varphi_{0}\right\rangle \text {. }
$$

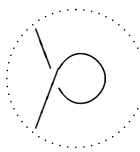

$\varphi_{+}$

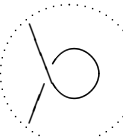

$\varphi_{-}$

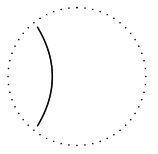

$\varphi_{0}$

FiguRE 7 
For a link $L$ with its diagram $D$, the Jones polynomial $V_{L}(t)$ of $L$ is defined as

$$
V_{L}(t)=(-A)^{-3 w(D)}\langle D\rangle
$$

by setting $A^{-4}=t[8]$.

Lemma 3.1. For each integer $n$, let $T(n)$ be a diagram with $n$-half twists and fixed outside as described in Figure 8. Then for any integer $n \geq 1$, we have

$$
\langle T(n)\rangle=A^{-n}\langle T(0)\rangle+A^{-n+2} \sum_{i=0}^{n-1}\left(-A^{4}\right)^{i}\langle T(\infty)\rangle
$$

and

$$
\langle T(-n)\rangle=A^{n}\langle T(0)\rangle+A^{n-2} \sum_{i=0}^{n-1}\left(-A^{-4}\right)^{i}\langle T(\infty)\rangle .
$$

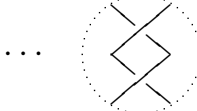

$T(-2)$

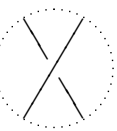

$T(-1)$

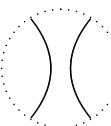

$T(0)$

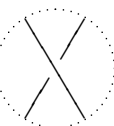

$T(1)$

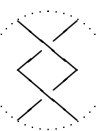

$T(2)$

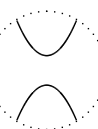

$T(\infty)$

Figure 8

Proof. First we will prove that the equation (6) holds. If $n=1$, then $\langle T(1)\rangle=$ $A^{-1}\langle T(0)\rangle+A\langle T(\infty)\rangle$ by (3). For a positive integer $n>1$, we assume that

$$
\langle T(n)\rangle=A^{-n}\langle T(0)\rangle+A^{-n+2} \sum_{i=0}^{n-1}\left(-A^{4}\right)^{i}\langle T(\infty)\rangle .
$$

Then it follows that

$$
\begin{aligned}
\langle T(n+1)\rangle= & A^{-1}\langle T(n)\rangle+A(-A)^{3 n}\langle T(\infty)\rangle \\
= & A^{-1}\left(A^{-n}\langle T(0)\rangle+A^{-n+2} \sum_{i=0}^{n-1}\left(-A^{4}\right)^{i}\langle T(\infty)\rangle\right) \\
& +A^{-(n+1)+2}\left(-A^{4}\right)^{n}\langle T(\infty)\rangle \\
= & A^{-(n+1)}\langle T(0)\rangle+A^{-(n+1)+2} \sum_{i=0}^{n}\left(-A^{4}\right)^{i}\langle T(\infty)\rangle .
\end{aligned}
$$

By a similar argument, we obtain the equation (7). 
For any nonzero integer $n$, we define Laurent polynomials $\alpha_{n}$ and $\beta_{n}$ by

$$
\alpha_{n}=A^{-n}, \quad \beta_{n}=\left\{\begin{array}{cl}
A^{-n+2} \sum_{i=0}^{n-1}\left(-A^{4}\right)^{i} & \text { if } n \geq 1, \\
A^{-n-2} \sum_{i=0}^{-n-1}\left(-A^{-4}\right)^{i} & \text { if } n \leq-1 .
\end{array}\right.
$$

Then we have easily:

Lemma 3.2. For given nonzero integers $n$ and $p$, we have that

$$
\beta_{n} \delta+p \alpha_{n}=A^{-n}\left(\left(-A^{4}\right)^{n}+(p-1)\right) .
$$

For any nonzero integers $n_{1}, n_{2}, \ldots, n_{r}(r \geq 1)$ and a positive integer $p \geq 2$, let $L^{(p)}$ be the $p$-periodic link in $S^{3}$ with rational quotient $L=\vec{C}\left[\left[n_{1}, n_{2}, \ldots\right.\right.$, $\left.n_{r}\right]$ ]. We consider the $p$-periodic diagram $D^{(p)}$ of $L^{(p)}$ as shown in Figure 9 . In Figure 9, each $T_{i, j}$ denotes a 2-tangle with $n_{i}$-half twists as in Figure 3 . If $n_{i}$ is positive (respectively, negative), each crossing of $T_{i, j}$ is positive (respectively, negative). Since the writhe, denoted by $w\left(D^{(p)}\right)$, of $D^{(p)}$ is the sum of crossing signs of crossings in $D^{(p)}$, we get

$$
w\left(D^{(p)}\right)=p \sum_{i=1}^{r} n_{i} .
$$

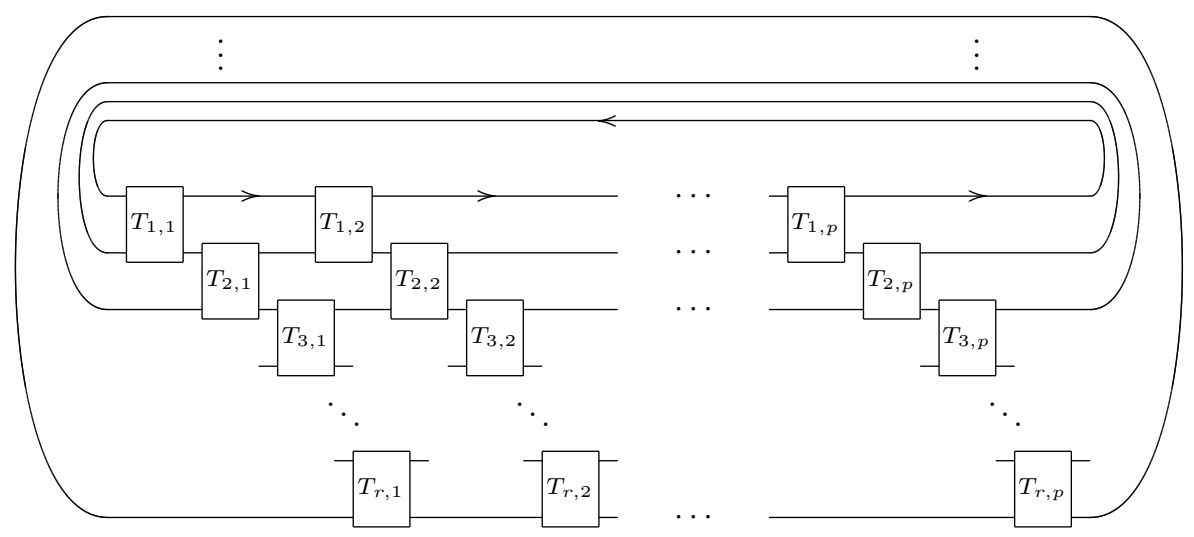

FIGURE 9

Put $\mathcal{T}_{i}=\left\{T_{i, 1}, T_{i, 2}, \ldots, T_{i, p}\right\}$ for each $i=1,2, \ldots, r$. We call a function $s: \mathcal{T}_{i} \rightarrow\{0, \infty\}$, where 0 denotes $T(0)$ and $\infty$ denotes $T(\infty)$ a weight function of $\mathcal{T}_{i}$. For each $i=1,2, \ldots, r$, let $\mathcal{S}_{i}$ denote the set of all weight functions of $\mathcal{T}_{i}$. For a weight function $s \in \mathcal{S}_{r}$, let $D^{(p)}(s)$ be the diagram obtained from $D^{(p)}$ by replacing each tangle $T_{r, k}$ in $\mathcal{T}_{r}$ by a $s\left(T_{r, k}\right)$-tangle and we denote by $\phi(s)$ the number of the tangles in $s^{-1}(0)$. By applying Lemma 3.1 to each tangle $T_{r, k}$ in $\mathcal{T}_{r}$, we have: 
Proposition 3.3. For given nonzero integers $n_{1}, n_{2}, \ldots, n_{r}(r \geq 1)$ and a positive integer $p \geq 2$, let $L^{(p)}$ be the $p$-periodic link with rational quotient $L=\vec{C}\left[\left[n_{1}, n_{2}, \ldots, n_{r}\right]\right]$ and $D^{(p)}$ its $p$-periodic diagram as shown in Figure 9. Then

$$
\left\langle D^{(p)}\right\rangle=\sum_{s \in \mathcal{S}_{r}} \alpha_{n_{r}}^{\phi(s)} \beta_{n_{r}}^{p-\phi(s)}\left\langle D^{(p)}(s)\right\rangle .
$$

For an $r$-tuple $\left(s_{1}, s_{2}, \ldots, s_{r}\right)$ of weight functions with $s_{i} \in \mathcal{S}_{i}(i=1,2, \ldots, r)$, let $D^{(p)}\left(s_{1}, \ldots, s_{r}\right)$ be the diagram obtained from $D^{(P)}$ by replacing each tangle $T_{i, j}$ with the $s_{i}\left(T_{i, j}\right)$-tangle and we denote by $\phi\left(s_{k}\right)$ the number of tangles in $s_{k}^{-1}(0)$ for each $k=1,2, \ldots, r$. By applying Lemma 3.1 to each tangle $T_{i, j}$ in $D^{(p)}$, we also have:

Proposition 3.4. For given nonzero integers $n_{1}, n_{2}, \ldots, n_{r}(r \geq 1)$ and a positive integer $p \geq 2$, let $L^{(p)}$ be the $p$-periodic link with rational quotient $L=\vec{C}\left[\left[n_{1}, n_{2}, \ldots, n_{r}\right]\right]$ and $D^{(p)}$ its $p$-periodic diagram as shown in Figure 9. Then

$$
\left\langle D^{(p)}\right\rangle=\sum_{\left(s_{1}, \ldots, s_{r}\right) \in \mathcal{S}_{1} \times \cdots \times \mathcal{S}_{r}}\left(\prod_{k=1}^{r} \alpha_{n_{k}}^{\phi\left(s_{k}\right)} \beta_{n_{k}}^{p-\phi\left(s_{k}\right)}\right) \delta^{\left|D^{(p)}\left(s_{1}, \ldots, s_{r}\right)\right|-1},
$$

where $\delta=-A^{2}-A^{-2}$ and $\left|D^{(p)}\left(s_{1}, \ldots, s_{r}\right)\right|$ is the number of disjoint simple closed curves in $D^{(p)}\left(s_{1}, \ldots, s_{r}\right)$.

Remark 3.5. Each diagram $D^{(p)}\left(s_{1}, \ldots, s_{r}\right)$ is a disjoint union of simple closed curves. If we can find a formula for the number of disjoint simple closed curves in each $D^{(p)}\left(s_{1}, \ldots, s_{r}\right)$ in terms of $n_{1}, n_{2}, \ldots, n_{r}$ and $p$, then the Laurent polynomial $\left\langle D^{(p)}\right\rangle$ can be expressed by means of the integers $n_{1}, n_{2}, \ldots, n_{r}$ and $p$. However, it looks very difficult to make such a formula. The authors know of none.

\section{Recursive formula for the Jones polynomial of 2-bridge links}

It is well known that any 2-bridge knot or link admits a diagram with Conway normal form $C\left(2 a_{1}, 2 a_{2}, \ldots, 2 a_{r}\right)$ for some integers $a_{1}, a_{2}, \ldots, a_{r}$ [7]. In [3], Jang, the second and third authors proved that the 2 -periodic link $L^{(2)}$ with rational quotient $L=\vec{C}\left[\left[n_{1}, n_{2}, \ldots, n_{k}\right]\right]$ is a 2-bridge knot or link with Conway normal form $C\left(-2 n_{1}, 2 n_{2},-2 n_{3}, \ldots,(-1)^{r} 2 n_{r}\right)$. In this section we give a recursive formula for the Jones polynomial of a 2-periodic link with rational quotient and give a formula for the span of the Jones polynomial. Consequently, we get a recursive formula for the Jones polynomial of 2-bridge knot or link with Conway normal form $C\left(2 a_{1}, 2 a_{2}, \ldots, 2 a_{r}\right)$ in terms of $a_{1}, a_{2}, \ldots, a_{r}$.

Lemma 4.1. Let $n_{1}, n_{2}, \ldots, n_{r}$ be given nonzero integers. For each $k=$ $1,2, \ldots, r$, let $D_{k}^{(2)}$ be the canonical 2-periodic diagram of the 2-periodic link 
with rational quotient $L_{k}=\vec{C}\left[\left[n_{1}, n_{2}, \ldots, n_{k}\right]\right]$. Let $D_{0}^{(2)}$ denote the standard diagram of the unknot. Then we have the following recursive formula:

$$
\begin{aligned}
\left\langle D_{0}^{(2)}\right\rangle & =1 \\
\left\langle D_{1}^{(2)}\right\rangle & =\beta_{n_{1}}^{2} \delta+2 \alpha_{n_{1}} \beta_{n_{1}}+\alpha_{n_{1}}^{2} \delta \\
\left\langle D_{k}^{(2)}\right\rangle & =\left(\beta_{n_{k}}^{2} \delta+2 \alpha_{n_{k}} \beta_{n_{k}}\right)\left\langle D_{k-1}^{(2)}\right\rangle+\alpha_{n_{k}}^{2} A^{6 n_{k-1}}\left\langle D_{k-2}^{(2)}\right\rangle .
\end{aligned}
$$

Proof. For a weight function $s \in \mathcal{S}_{k}$, let $D_{k}^{(2)}\left(s\left(T_{k, 1}\right), s\left(T_{k, 2}\right)\right)$ be the diagram obtained from $D_{k}^{(2)}$ by replacing each tangle $T_{k, i}$ by an $s\left(T_{k, i}\right)$-tangle $(i=1,2)$.

If $k=1$, then $D_{1}^{(2)}(0,0), D_{1}^{(2)}(0, \infty), D_{1}^{(2)}(\infty, 0)$ and $D_{1}^{(2)}(\infty, \infty)$ consist of simple closed curves without crossings. We observe that $D_{1}^{(2)}(0,0)$ and $D_{1}^{(2)}(\infty, \infty)$ have two components and $D_{1}^{(2)}(0, \infty)$ and $D_{1}^{(2)}(\infty, 0)$ have one component. By Proposition 3.4, we have

$$
\left\langle D_{1}^{(2)}\right\rangle=\beta_{n_{1}}^{2} \delta+2 \alpha_{n_{1}} \beta_{n_{1}}+\alpha_{n_{1}}^{2} \delta .
$$

Now we assume that the recursive formula (9) holds for $n_{1}, n_{2}, \ldots, n_{k-1}$ with $k \geq 2$. Then $D_{k}^{(2)}(0,0), D_{k}^{(2)}(0, \infty), D_{k}^{(2)}(\infty, 0)$ and $D_{k}^{(2)}(\infty, \infty)$ are isotopic to the diagrams as shown in Figure 10 . Thus $D_{k}^{(2)}(0, \infty)$ and $D_{k}^{(2)}(\infty, 0)$ are

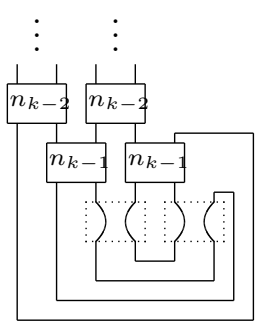

$D_{k}^{(2)}(0,0)$

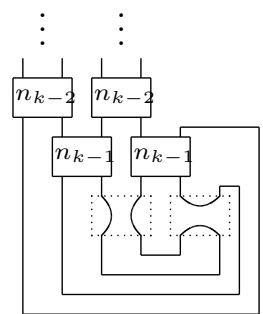

$D_{k}^{(2)}(0, \infty)$

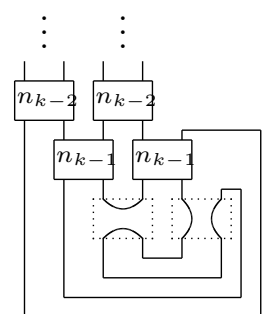

$D_{k}^{(2)}(\infty, 0)$

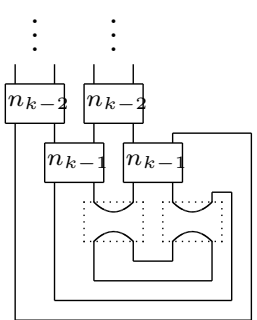

$D_{k}^{(2)}(\infty, \infty)$

FIGURE 10

isotopic to the diagram $D_{k-1}^{(2)}$, and $D_{k}^{(2)}(\infty, \infty)$ is isotopic to the diagram $D_{k-1}^{(2)} \sqcup$ O. Moreover $D_{k}^{(2)}(0,0)$ is obtained from $D_{k-2}^{(2)}$ by applying the Reidemeister move I. By (2), (5) and Proposition 3.3, we have

$$
\begin{aligned}
\left\langle D_{k}^{(2)}\right\rangle= & \beta_{k}^{2}\left\langle D_{k}^{(2)}(\infty, \infty)\right\rangle+\beta_{k} \alpha_{k}\left\langle D_{k}^{(2)}(\infty, 0)\right\rangle \\
& +\alpha_{k} \beta_{k}\left\langle D_{k}^{(2)}(0, \infty)\right\rangle+\alpha_{k}^{2}\left\langle D_{k}^{(2)}(0,0)\right\rangle \\
= & \left(\beta_{k}^{2} \delta+2 \alpha_{k} \beta_{k}\right)\left\langle D_{k-1}^{(2)}\right\rangle+\alpha_{k}^{2} A^{6 n_{k-1}}\left\langle D_{k-2}^{(2)}\right\rangle .
\end{aligned}
$$

This completes the proof. 
For any nonzero integer $n$, let $\mathcal{A}_{n}(t)$ be a Laurent polynomial in $\mathbb{Z}\left[t^{ \pm \frac{1}{2}}\right]$ defined by

$$
\mathcal{A}_{n}(t)= \begin{cases}t^{-\frac{1}{2}} \sum_{i=0}^{n-1}(-t)^{-i} & \text { if } n \geq 1, \\ t^{\frac{1}{2}} \sum_{i=0}^{-n-1}(-t)^{i} & \text { if } n \leq-1 .\end{cases}
$$

We note that $\left.\beta_{n}\right|_{A=t^{-\frac{1}{4}}}=t^{\frac{n}{4}} \mathcal{A}_{n}(t)$.

Theorem 4.2. Let $n_{1}, n_{2}, \ldots, n_{r}$ be given nonzero integers. For each $k=$ $1,2, \ldots, r$, let $L_{k}^{(2)}$ be the 2-periodic link with rational quotient $L_{k}=\vec{C}\left[\left[n_{1}, n_{2}\right.\right.$, $\left.\left.\ldots, n_{k}\right]\right]$ and let $L_{0}^{(2)}$ the trivial knot. Let $V_{k}(t)$ be the Jones polynomial of $L_{k}^{(2)}$ for each $k=0,1,2, \ldots, r$. Then we have the following recursive formula:

$$
\begin{aligned}
& V_{0}(t)=1, \\
& V_{1}(t)=t^{2 n_{1}}\left(\mathcal{A}_{2 n_{1}}(t)-t^{\frac{1}{2}}-t^{-\frac{1}{2}}\right), \\
& V_{k}(t)=t^{2 n_{k}} \mathcal{A}_{2 n_{k}}(t) V_{k-1}(t)+t^{2 n_{k}} V_{k-2}(t) .
\end{aligned}
$$

Proof. For each $k=1,2, \ldots, r$, let $D_{k}^{(2)}$ be the canonical 2-periodic diagram of the 2-periodic link $L_{k}^{(2)}$. Then

$$
V_{k}(t)=\left.(-A)^{-3 w\left(D_{k}^{(2)}\right)}\left\langle D_{k}^{(2)}\right\rangle\right|_{A=t^{-\frac{1}{4}}} .
$$

For each $k=1,2, \ldots, r$, put $f_{k}(A)=(-A)^{-3 w\left(D_{k}^{(2)}\right)}\left\langle D_{k}^{(2)}\right\rangle$. Then $V_{k}(t)=$ $\left.f_{k}(A)\right|_{A=t^{-\frac{1}{4}}}$. We note that $w\left(D_{k}^{(2)}\right)=2 \sum_{i=1}^{k} n_{i}$ and, by Lemma $3.2, \beta_{n_{i}} \delta+$ $2 \alpha_{n_{i}}=A^{-n_{i}}\left(\left(-A^{4}\right)^{n_{i}}+1\right)$.

Since $L_{0}^{(2)}$ is the trivial knot, $V_{0}(t)=1$. If $n_{1} \geq 1$, then

$$
\begin{aligned}
f_{1}(A) & =(-A)^{-6 n_{1}}\left(\beta_{n_{1}}\left(\beta_{n_{1}} \delta+2 \alpha_{n_{1}}\right)+\alpha_{n_{1}}^{2} \delta\right) \\
& =A^{-6 n_{1}}\left(A^{-2 n_{1}+2}\left(\left(-A^{4}\right)^{n_{1}}+1\right) \sum_{i=0}^{n_{1}-1}\left(-A^{4}\right)^{i}+A^{-2 n_{1}}\left(-A^{2}-A^{-2}\right)\right) \\
& =A^{-8 n_{1}}\left(A^{2} \sum_{i=0}^{2 n_{1}-1}\left(-A^{4}\right)^{i}+\left(-A^{2}-A^{-2}\right)\right)
\end{aligned}
$$

and hence

$$
V_{1}(t)=t^{2 n_{1}}\left(t^{-\frac{1}{2}} \sum_{i=0}^{2 n_{1}-1}(-t)^{-i}+\left(-t^{-\frac{1}{2}}-t^{\frac{1}{2}}\right)\right)=t^{2 n_{1}}\left(\mathcal{A}_{2 n_{1}}(t)-t^{\frac{1}{2}}-t^{-\frac{1}{2}}\right) .
$$


If $n_{1} \leq-1$, then $f_{1}(A)=A^{-8 n_{1}}\left(A^{-2} \sum_{i=0}^{-2 n_{1}-1}\left(-A^{-4}\right)^{i}+\left(-A^{2}-A^{-2}\right)\right)$ and hence

$$
V_{1}(t)=t^{2 n_{1}}\left(t^{\frac{1}{2}} \sum_{i=0}^{-2 n_{1}-1}(-t)^{i}+\left(-t^{-\frac{1}{2}}-t^{\frac{1}{2}}\right)\right)=t^{2 n_{1}}\left(\mathcal{A}_{2 n_{1}}(t)-t^{\frac{1}{2}}-t^{-\frac{1}{2}}\right)
$$

For $k \geq 2$, from Lemma 4.1, we obtain

$$
f_{k}(A)=(-A)^{-6 n_{k}}\left(\beta_{n_{k}}^{2} \delta+2 \alpha_{n_{k}} \beta_{n_{k}}\right) f_{k-1}(A)+(-A)^{-6 n_{k}} \alpha_{n_{k}}^{2} f_{k-2}(A) .
$$

Immediately we have $\left.(-A)^{-6 n_{k}} \alpha_{n_{k}}^{2}\right|_{A=t^{-\frac{1}{4}}}=t^{2 n_{k}}$. If $n_{k} \geq 1$, then it follows that

and hence

$$
(-A)^{-6 n_{k}}\left(\beta_{n_{k}}^{2} \delta+2 \alpha_{n_{k}} \beta_{n_{k}}\right)=A^{-8 n_{k}+2} \sum_{i=0}^{2 n_{k}-1}\left(-A^{4}\right)^{i}
$$

$$
\left.(-A)^{-6 n_{k}}\left(\beta_{n_{k}}^{2} \delta+2 \alpha_{n_{k}} \beta_{n_{k}}\right)\right|_{A=t^{-\frac{1}{4}}}=t^{2 n_{k}} \mathcal{A}_{2 n_{k}}(t) .
$$

If $n_{k} \leq-1$, then

$$
(-A)^{-6 n_{k}}\left(\beta_{n_{k}}^{2} \delta+2 \alpha_{n_{k}} \beta_{n_{k}}\right)=A^{-8 n_{k}-2} \sum_{i=0}^{-2 n_{k}-1}\left(-A^{-4}\right)^{i}
$$

and hence

$$
\left.(-A)^{-6 n_{k}}\left(\beta_{n_{k}}^{2} \delta+2 \alpha_{n_{k}} \beta_{n_{k}}\right)\right|_{A=t^{-\frac{1}{4}}}=t^{2 n_{k}} \mathcal{A}_{2 n_{k}}(t) .
$$

Therefore we have

$$
\begin{aligned}
V_{k}(t) & =\left.f_{k}(A)\right|_{A=t^{-\frac{1}{4}}} \\
& =\left.A^{-6 n_{k}}\left(\beta_{n_{k}}^{2} \delta+2 \alpha_{n_{k}} \beta_{n_{k}}\right)\right|_{A=t^{-\frac{1}{4}}} V_{k-1}(t)+\left.A^{-6 n_{k}} \alpha_{n_{k}}^{2}\right|_{A=t^{-\frac{1}{4}}} V_{k-2}(t) \\
& =t^{2 n_{k}} \mathcal{A}_{2 n_{k}}(t) V_{k-1}(t)+t^{2 n_{k}} V_{k-2}(t) .
\end{aligned}
$$

This completes the proof.

Example 4.3. Let $L$ be the 2-bridge knot with Conway normal form $C(-2$, $-4)$. It is the mirror image of the knot $5_{2}$ in Rolfsen's table [20]. By the discussion in the beginning of this section, $L$ is the 2-periodic knot with rational quotient $\vec{C}[[1,2]]$. Let $n_{1}=1$ and $n_{2}=2$. Then we have that $\mathcal{A}_{2}(t)=t^{-\frac{1}{2}}-t^{-\frac{3}{2}}$ and $\mathcal{A}_{4}(t)=t^{-\frac{1}{2}}-t^{-\frac{3}{2}}+t^{-\frac{5}{2}}-t^{-\frac{7}{2}}$. From Theorem 4.2, it follows that $V_{0}(t)=1$, $V_{1}(t)=-t^{\frac{1}{2}}-t^{\frac{5}{2}}$ and $V_{2}(t)=-t^{6}+t^{5}-t^{4}+2 t^{3}-t^{2}+t$. Hence the Jones polynomial of $L$ is

$$
V_{L}(t)=-t^{6}+t^{5}-t^{4}+2 t^{3}-t^{2}+t .
$$

For the Jones polynomial $V_{L}(t)$ of a link $L$, we denote the maximum (resp. minimum) degree of $V_{L}(t)$ by $\max \operatorname{deg} V_{L}(t)$ (resp. min $\operatorname{deg} V_{L}(t)$ ). We also denote the span of $V_{L}(t)$ by span $V_{L}(t)$, i.e., span $V_{L}(t)=\max \operatorname{deg} V_{L}(t)-$ $\min \operatorname{deg} V_{L}(t)$. 
Lemma 4.4. For given nonzero integers $n_{1}, n_{2}, \ldots, n_{r}$, let $V_{k}(t)$ be the Jones polynomial of the 2-periodic link $L_{k}^{(2)}$ with rational quotient $L=\vec{C}\left[\left[n_{1}, n_{2}\right.\right.$, $\left.\left.\ldots, n_{k}\right]\right]$ for each $k=1,2, \ldots, r$. Put $\epsilon_{k}=\frac{\left|n_{k}\right|}{n_{k}}(k=1,2, \ldots, r)$. For each $k=1,2, \ldots, r$, we have that

(14) $\max \operatorname{deg} V_{k}(t)=\frac{1-k}{2}+\sum_{i=1}^{k}\left(n_{i}+\left|n_{i}\right|\right)+\frac{\epsilon_{1}}{2}+\frac{1}{4} \sum_{j=1}^{k-1}\left(1-\epsilon_{j}\right)\left(1+\epsilon_{j+1}\right)$

and

(15) $\min \operatorname{deg} V_{k}(t)=\frac{k-1}{2}+\sum_{i=1}^{k}\left(n_{i}-\left|n_{i}\right|\right)+\frac{\epsilon_{1}}{2}-\frac{1}{4} \sum_{j=1}^{k-1}\left(1+\epsilon_{j}\right)\left(1-\epsilon_{j+1}\right)$.

Proof. Let $n$ be any nonzero integer. From (10), we have that

$$
\max \operatorname{deg} \mathcal{A}_{2 n}(t)=-\frac{1}{2}+|n|-n, \quad \min \operatorname{deg} \mathcal{A}_{2 n}(t)=\frac{1}{2}-|n|-n .
$$

We will use the recursive formula in Theorem 4.2 and induction on $k$.

If $n_{1} \geq 1$, then max $\operatorname{deg} \mathcal{A}_{2 n_{1}}(t)=-\frac{1}{2}$ and hence max $\operatorname{deg} V_{1}(t)=2 n_{1}+\frac{1}{2}$. If $n_{1} \leq-1$, then max $\operatorname{deg} \mathcal{A}_{2 n_{1}}(t)=-\frac{1}{2}-2 n_{1}$ and hence $\max \operatorname{deg} V_{1}(t) \stackrel{=}{=}$ $2 n_{1}-\frac{1}{2}-2 n_{1}=-\frac{1}{2}$. Therefore we have

$$
\max \operatorname{deg} V_{1}(t)=\left(n_{1}+\left|n_{1}\right|\right)+\frac{\epsilon_{1}}{2} .
$$

We assume that the formula (14) holds for $k$.

Case (i) : If $n_{k+1} \leq-1$ or $n_{k} \geq 1$, then

$$
\begin{aligned}
& \max \operatorname{deg} \mathcal{A}_{2 n_{k+1}}(t) V_{k}(t) \\
= & \max \operatorname{deg} \mathcal{A}_{2 n_{k+1}}(t)+\max \operatorname{deg} V_{k}(t) \\
= & -\frac{1}{2}+\left|n_{k+1}\right|-n_{k+1} \\
& +\frac{1-k}{2}+\sum_{i=1}^{k}\left(n_{i}+\left|n_{i}\right|\right)+\frac{\epsilon_{1}}{2}+\frac{1}{4} \sum_{j=1}^{k-1}\left(1-\epsilon_{j}\right)\left(1+\epsilon_{j+1}\right) \\
\geq & \frac{2-k}{2}+\sum_{i=1}^{k-1}\left(n_{i}+\left|n_{i}\right|\right)+\frac{\epsilon_{1}}{2}+\frac{1}{4} \sum_{j=1}^{k-2}\left(1-\epsilon_{j}\right)\left(1+\epsilon_{j+1}\right) \\
& -1+\left|n_{k+1}\right|-n_{k+1}+\left(n_{k}+\left|n_{k}\right|\right)
\end{aligned}
$$

and

$$
\max \operatorname{deg} V_{k-1}(t)=\frac{2-k}{2}+\sum_{i=1}^{k-1}\left(n_{i}+\left|n_{i}\right|\right)+\frac{\epsilon_{1}}{2}+\frac{1}{4} \sum_{j=1}^{k-2}\left(1-\epsilon_{j}\right)\left(1+\epsilon_{j+1}\right) .
$$


Since $-1+\left|n_{k+1}\right|-n_{k+1}+\left(n_{k}+\left|n_{k}\right|\right) \geq 1$,

$$
\max \operatorname{deg} \mathcal{A}_{2 n_{k+1}}(t) V_{k}(t)>\max \operatorname{deg} V_{k-1}(t) .
$$

Thus by (13), we have that

$$
\begin{aligned}
& \max \operatorname{deg} V_{k+1}(t) \\
= & 2 n_{k+1}+\max \operatorname{deg} \mathcal{A}_{2 n_{k+1}}(t) V_{k}(t) \\
= & 2 n_{k+1}-\frac{1}{2}+\left|n_{k+1}\right|-n_{k+1} \\
& +\frac{1-k}{2}+\sum_{i=1}^{k}\left(n_{i}+\left|n_{i}\right|\right)+\frac{\epsilon_{1}}{2}+\frac{1}{4} \sum_{j=1}^{k-1}\left(1-\epsilon_{j}\right)\left(1+\epsilon_{j+1}\right) \\
= & \frac{1-(k+1)}{2}+\sum_{i=1}^{k+1}\left(n_{i}+\left|n_{i}\right|\right)+\frac{\epsilon_{1}}{2}+\frac{1}{4} \sum_{j=1}^{k}\left(1-\epsilon_{j}\right)\left(1+\epsilon_{j+1}\right) .
\end{aligned}
$$

Case (ii) : If $n_{k+1} \geq 1$ and $n_{k} \leq-1$, then

$$
\begin{aligned}
& \max \operatorname{deg} \mathcal{A}_{2 n_{k+1}}(t) V_{k}(t) \\
= & \max \operatorname{deg} \mathcal{A}_{2 n_{k+1}}(t)+\max \operatorname{deg} V_{k}(t) \\
= & -\frac{1}{2}+\frac{1-k}{2}+\sum_{i=1}^{k-1}\left(n_{i}+\left|n_{i}\right|\right)+\frac{\epsilon_{1}}{2}+\frac{1}{4} \sum_{j=1}^{k-2}\left(1-\epsilon_{j}\right)\left(1+\epsilon_{j+1}\right)
\end{aligned}
$$

and

$$
\max \operatorname{deg} V_{k-1}(t)=\frac{2-k}{2}+\sum_{i=1}^{k-1}\left(n_{i}+\left|n_{i}\right|\right)+\frac{\epsilon_{1}}{2}+\frac{1}{4} \sum_{j=1}^{k-2}\left(1-\epsilon_{j}\right)\left(1+\epsilon_{j+1}\right) .
$$

Hence $\max \operatorname{deg} \mathcal{A}_{2 n_{k+1}}(t) V_{k}(t)+1=\max \operatorname{deg} V_{k-1}(t)$. Thus we have

$$
\begin{aligned}
& \max \operatorname{deg} V_{k+1}(t) \\
= & 2 n_{k+1}+\max \operatorname{deg} V_{k-1}(t) \\
= & 2 n_{k+1}+\frac{2-k}{2}+\sum_{i=1}^{k-1}\left(n_{i}+\left|n_{i}\right|\right)+\frac{\epsilon_{1}}{2}+\frac{1}{4} \sum_{j=1}^{k-2}\left(1-\epsilon_{j}\right)\left(1+\epsilon_{j+1}\right) \\
= & \frac{1-(k+1)}{2}+\sum_{i=1}^{k+1}\left(n_{i}+\left|n_{i}\right|\right)+\frac{\epsilon_{1}}{2}+\frac{1}{4} \sum_{j=1}^{k}\left(1-\epsilon_{j}\right)\left(1+\epsilon_{j+1}\right) .
\end{aligned}
$$

By a similar argument, we also have the formula (15).

For given nonzero integers $n_{1}, n_{2}, \ldots, n_{r}$, we define an integer $\kappa\left(n_{1}, n_{2}\right.$, $\ldots, n_{r}$ ) (or briefly $\kappa\left(n_{i} ; r\right)$ ) as the number of elements in the $\operatorname{set}\left\{\left(n_{i}, n_{i+1}\right)\right.$ | $\left.n_{i} n_{i+1}>0,1 \leq i \leq r-1\right\}$. For example, $\kappa(2,3,2,-1)=2, \kappa(1,2,3,4)=3$ and $\kappa(-1,1,-2,4)=0$. We note that $0 \leq \kappa\left(n_{i} ; r\right) \leq r-1$. 
Theorem 4.5. For given nonzero integers $n_{1}, n_{2}, \ldots, n_{r}$, let $L^{(2)}$ be the 2periodic link with rational quotient $L=\vec{C}\left[\left[n_{1}, n_{2}, \ldots, n_{r}\right]\right]$. Then the span of the Jones polynomial $V_{L^{(2)}}(t)$ of $L^{(2)}$ is given by

$$
\operatorname{span} V_{L^{(2)}}(t)=2 \sum_{i=1}^{r}\left|n_{i}\right|-\kappa\left(n_{i} ; r\right) \text {. }
$$

Proof. From Lemma 4.4, we have

$$
\begin{aligned}
& \operatorname{span} V_{L^{(2)}}(t)=\max \operatorname{deg} V_{L^{(2)}}(t)-\min \operatorname{deg} V_{L^{(2)}}(t) \\
= & \frac{1-r}{2}+\sum_{i=1}^{r}\left(n_{i}+\left|n_{i}\right|\right)+\frac{\epsilon_{1}}{2}+\frac{1}{4} \sum_{j=1}^{r-1}\left(1-\epsilon_{j}\right)\left(1+\epsilon_{j+1}\right) \\
& -\frac{r-1}{2}-\sum_{i=1}^{r}\left(n_{i}-\left|n_{i}\right|\right)-\frac{\epsilon_{1}}{2}+\frac{1}{4} \sum_{j=1}^{r-1}\left(1+\epsilon_{j}\right)\left(1-\epsilon_{j+1}\right) \\
= & (1-r)+2 \sum_{i=1}^{r}\left|n_{i}\right|+\frac{1}{4} \sum_{j=1}^{r-1}\left[\left(1-\epsilon_{j}\right)\left(1+\epsilon_{j+1}\right)+\left(1+\epsilon_{j}\right)\left(1-\epsilon_{j+1}\right)\right] \\
= & 2 \sum_{i=1}^{r}\left|n_{i}\right|-\left\{(r-1)-\sum_{j=1}^{r-1} \frac{1-\epsilon_{j} \epsilon_{j+1}}{2}\right\}
\end{aligned}
$$

Because $\left(1-\epsilon_{j} \epsilon_{j+1}\right) / 2$ is 1 if $n_{j}$ and $n_{j+1}$ have different signs and 0 otherwise, $\sum_{j=1}^{r-1}\left(1-\epsilon_{j} \epsilon_{j+1}\right) / 2$ counts the number of pairs $\left(n_{j}, n_{j+1}\right)$ with $n_{j} n_{j+1}<0$. Therefore,

$$
\kappa\left(n_{i} ; r\right)=(r-1)-\sum_{j=1}^{r-1} \frac{1-\epsilon_{j} \epsilon_{j+1}}{2}
$$

hence we have

$$
\operatorname{span} V_{L^{(2)}}(t)=2 \sum_{i=1}^{r}\left|n_{i}\right|-\kappa\left(n_{i} ; r\right) .
$$

This completes the proof.

Corollary 4.6. For given nonzero integers $a_{1}, a_{2}, \ldots, a_{r}$, let $L$ be the 2-bridge knot or link with Conway normal form $C\left(2 a_{1}, 2 a_{2}, \ldots, 2 a_{r}\right)$. Then the crossing number of $L$ is given by

$$
c(L)=2 \sum_{i=1}^{r}\left|a_{i}\right|-\kappa\left(-a_{1}, a_{2},-a_{3}, \ldots,(-1)^{r} a_{r}\right) .
$$

Proof. From [3, Theorem 2.1], $L$ is a 2-periodic link with rational quotient $\vec{C}\left[\left[-a_{1}, a_{2}, \ldots,(-1)^{r} a_{r}\right]\right]$ (for more detail, see Remark $4.7(2)$ ). By Theorem 4.5 , we obtain that

$$
\operatorname{span} V_{L}(t)=2 \sum_{i=1}^{r}\left|a_{i}\right|-\kappa\left(-a_{1}, a_{2},-a_{3}, \ldots,(-1)^{r} a_{r}\right) .
$$


Since any 2-bridge link is alternating, the span of its Jones polynomial is equal to its crossing number. Hence the crossing number of $L$ is given by

$$
c(L)=2 \sum_{i=1}^{r}\left|a_{i}\right|-\kappa\left(-a_{1}, a_{2},-a_{3}, \ldots,(-1)^{r} a_{r}\right) .
$$

This completes the proof.

Remark 4.7. (1) It should be noticed that the result in Corollary 4.6 is not new. It is well known that every 2-bridge knot or link $L$ has the standard Conway normal form $C\left(b_{1}, b_{2}, \ldots, b_{n}\right)$ such that all $b_{1}, b_{2}, \ldots, b_{n}$ are either positive or negative and $C\left(b_{1}, b_{2}, \ldots, b_{n}\right)$ is a reduced alternating diagram for $L$. Hence $c(L)=\left|b_{1}\right|+\left|b_{2}\right|+\cdots+\left|b_{n}\right|$. It is also known that $L$ admits a Conway normal form $C\left(2 a_{1}, 2 a_{2}, \ldots, 2 a_{r}\right)$ for some nonzero integers $a_{1}, a_{2}, \ldots, a_{r}$ [7], adopted in Corollary 4.6. The authors do not know whether the formula (17) of Corollary 4.6 can be directly derived from the standard Conway normal form $C\left(b_{1}, b_{2}, \ldots, b_{n}\right)$ or not.

(2) Let $L$ be a link of two components and let $L_{1}$ be the same link as $L$ but with the opposite orientation on only one component of $L$. Note that $L$ and $L_{1}$ are may be different. But the crossing numbers of $L$ and $L_{1}$ are the same. Since every 2-bridge link is invertible, there are at most two oriented 2-bridge links with the same unoriented diagram. Without loss of generality, in the proof of Corollary 4.6, we can consider that $L$ is a 2-periodic link with rational quotient $\vec{C}\left[\left[-a_{1}, a_{2}, \ldots,(-1)^{r} a_{r}\right]\right]$.

\section{Recursive formula for the Jones polynomial of 3-periodic links}

In this section, we give a recursive formula for the Jones polynomial of a 3 -periodic link with rational quotient. We also calculate the span of the Jones polynomial under certain conditions.

Lemma 5.1. Let $n_{1}, n_{2}, \ldots, n_{r}$ be given nonzero integers. For each $k=$ $1,2, \ldots, r$, let $D_{k}^{(3)}$ be the canonical 3-periodic diagram of the 3-periodic link with rational quotient $L_{k}=\vec{C}\left[\left[n_{1}, n_{2}, \ldots, n_{k}\right]\right]$. Let $D_{0}^{(2)}$ denote the standard diagram of the unknot. Then we have the following recursive formula:

$$
\begin{aligned}
\left\langle D_{0}^{(3)}\right\rangle= & 1 \\
\gamma_{1}= & \delta \\
\left\langle D_{1}^{(3)}\right\rangle= & 3 \alpha_{n_{1}} \beta_{n_{1}}^{2}+\left(3 \alpha_{n_{1}}^{2} \beta_{n_{1}}+\beta_{n_{1}}^{3}\right) \delta+\alpha_{n_{1}}^{3} \delta^{2} \\
\gamma_{k}= & (-A)^{3 n_{k-1}}\left(\alpha_{n_{k-1}}^{2} \gamma_{n_{k-1}}+\left(\beta_{n_{k-1}}^{2} \delta+2 \alpha_{n_{k-1}} \beta_{n_{k-1}}\right)\left\langle D_{k-2}^{(3)}\right\rangle\right) \\
\left\langle D_{k}^{(3)}\right\rangle= & \left(\beta_{n_{k}}^{3} \delta+3 \alpha_{n_{k}} \beta_{n_{k}}^{2}\right)\left\langle D_{k-1}^{(3)}\right\rangle+3 \alpha_{n_{k}}^{2} \beta_{n_{k}} \gamma_{k} \\
& +(-A)^{9 n_{k-1}} \alpha_{n_{k}}^{3}\left\langle D_{k-2}^{(3)}\right\rangle, \quad k=2,3, \ldots, r .
\end{aligned}
$$


Proof. For a weight function $s \in \mathcal{S}_{k}$, let $D_{k}^{(3)}\left(s\left(T_{k, 1}\right), s\left(T_{k, 2}\right), s\left(T_{k, 3}\right)\right)$ be the diagram obtained from $D_{k}^{(3)}$ by replacing each tangle $T_{k, i}$ by a $s\left(T_{k, i}\right)$-tangle $(i=1,2,3)$.

If $k=1$, then $D_{1}^{(3)}(0, \infty, \infty), D_{1}^{(3)}(\infty, 0, \infty)$ and $D_{1}^{(3)}(\infty, \infty, 0)$ consist of a simple closed curve. We also observe that $D_{1}^{(3)}(0,0, \infty), D_{1}^{(3)}(0, \infty, 0)$, $D_{1}^{(3)}(\infty, 0,0)$ and $D_{1}^{(3)}(\infty, \infty, \infty)$ consist of two simple closed curves, and $D_{1}^{(3)}(0,0,0)$ consists of three simple closed curves. By Proposition 3.4, we have

$$
\left\langle D_{1}^{(3)}\right\rangle=3 \alpha_{n_{1}} \beta_{n_{1}}^{2}+\left(3 \alpha_{n_{1}}^{2} \beta_{n_{1}}+\beta_{n_{1}}^{3}\right) \delta+\alpha_{n_{1}}^{3} \delta^{2} .
$$

Now we assume that the recursive formula (18) holds for $n_{1}, n_{2}, \ldots, n_{k-1}$ with $k \geq 2$. Then $D_{k}^{(3)}(\infty, \infty, \infty), D_{k}^{(3)}(0, \infty, \infty), D_{k}^{(3)}(\infty, 0, \infty), D_{k}^{(3)}(\infty, \infty, 0)$, $D_{k}^{(3)}(0,0, \infty), D_{k}^{(3)}(0, \infty, 0), D_{k}^{(3)}(\infty, 0,0)$ and $D_{k}^{(3)}(0,0,0)$ are isotopic to the diagrams as shown in Figure 11. Thus $D_{k}^{(3)}(0, \infty, \infty), D_{k}^{(3)}(\infty, 0, \infty)$ and $D_{k}^{(3)}(\infty, \infty, 0)$ are isotopic to the diagram $D_{k-1}^{(3)}$, and $D_{k}^{(3)}(\infty, \infty, \infty)$ is isotopic to the diagram $D_{k-1}^{(3)} \sqcup \bigcirc$. Moreover $D_{k}^{(3)}(0,0,0)$ is obtained from $D_{k-2}^{(3)}$ by applying the Reidemeister move I. Since $D_{k}^{(3)}$ is a periodic diagram, $D_{k}^{(3)}(0,0, \infty)$, $D_{k}^{(3)}(0, \infty, 0)$ and $D_{k}^{(3)}(\infty, 0,0)$ are isotopic to each other. Let $\overline{D_{k}^{(3)}(\infty, 0,0)}$ and $\overline{\overline{D_{k}^{(3)}(\infty, 0,0)}}$ be the diagrams in Figure $12(\mathrm{a})$ and (b), respectively. They are obtained from $D_{k-1}^{(3)}(\infty, 0,0)$ and $D_{k-2}^{(3)}$ respectively by applying the Reidemeister move I. For each $k=1,2, \ldots, r$, we define a Laurent polynomials $\gamma_{k}$ by

$$
\gamma_{k}=\left\langle D_{k}^{(3)}(\infty, 0,0)\right\rangle
$$

Since $D_{1}^{(3)}(\infty, 0,0)$ is of two components, $\gamma_{1}=\delta$. By applying Lemma 3.1 to $T_{k-1,1}$ and $T_{k-1,2}$ in $D_{k}^{(3)}(\infty, 0,0)$, we get

$$
\begin{aligned}
\gamma_{k} & =\alpha_{n_{k-1}}^{2}\left\langle\overline{\left.D_{k}^{(3)}(\infty, 0,0)\right\rangle}+\left(\beta_{n_{k-1}}^{2} \delta+2 \alpha_{n_{k-1}} \beta_{n_{k-1}}\right)\left\langle\overline{\overline{D_{k}^{(3)}(\infty, 0,0)}}\right.\right. \\
& =(-A)^{3 n_{k-1}}\left(\alpha_{n_{k-1}}^{2} \gamma_{n_{k-1}}+\left(\beta_{n_{k-1}}^{2} \delta+2 \alpha_{n_{k-1}} \beta_{n_{k-1}}\right)\left\langle D_{k-2}^{(3)}\right\rangle\right) .
\end{aligned}
$$

Hence we have

$$
\begin{aligned}
\left\langle D_{k}^{(3)}\right\rangle= & \beta_{n_{k}}^{3}\left\langle D_{k}^{(3)}(\infty, \infty, \infty)\right\rangle+\beta_{n_{k}}^{2} \alpha_{n_{k}}\left\langle D_{k}^{(3)}(0, \infty, \infty)\right\rangle \\
& +\beta_{n_{k}}^{2} \alpha_{n_{k}}\left\langle D_{k}^{(3)}(\infty, 0, \infty)\right\rangle+\beta_{n_{k}}^{2} \alpha_{n_{k}}\left\langle D_{k}^{(3)}(\infty, \infty, 0)\right\rangle \\
& +\beta_{n_{k}} \alpha_{n_{k}}^{2}\left\langle D_{k}^{(3)}(0,0, \infty)\right\rangle+\beta_{n_{k}} \alpha_{n_{k}}^{2}\left\langle D_{k}^{(3)}(0, \infty, 0)\right\rangle \\
& +\beta_{n_{k}} \alpha_{n_{k}}^{2}\left\langle D_{k}^{(3)}(\infty, 0,0)\right\rangle+\alpha_{n_{k}}^{3}\left\langle D_{k}^{(3)}(0,0,0)\right\rangle \\
= & \left(\beta_{n_{k}}^{3} \delta+3 \alpha_{n_{k}} \beta_{n_{k}}^{2}\right)\left\langle D_{k-1}^{(3)}\right\rangle+3 \alpha_{n_{k}}^{2} \beta_{n_{k}} \gamma_{k}+(-A)^{9 n_{k-1}} \alpha_{n_{k}}^{3}\left\langle D_{k-2}^{(3)}\right\rangle .
\end{aligned}
$$

This completes the proof. 


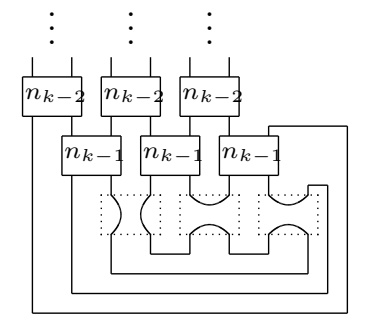

$$
D_{k}^{(3)}(0, \infty, \infty)
$$

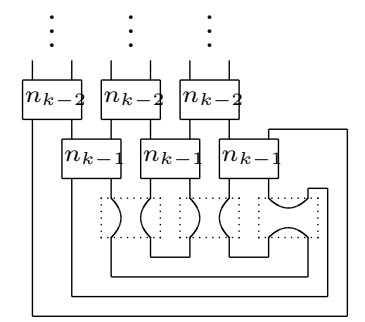

$D_{k}^{(3)}(0,0, \infty)$

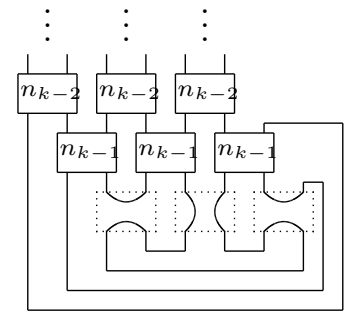

$D_{k}^{(3)}(\infty, 0, \infty)$

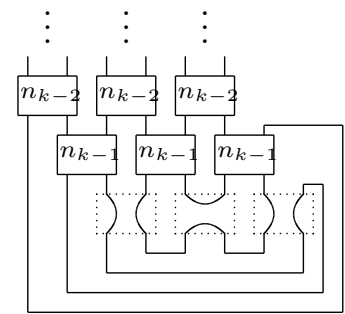

$D_{k}^{(3)}(0, \infty, 0)$

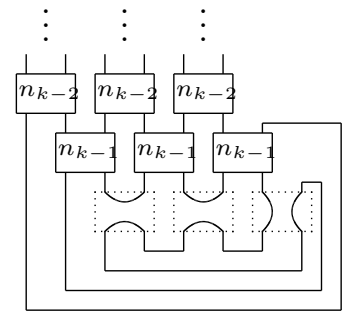

$D_{k}^{(3)}(\infty, \infty, 0)$

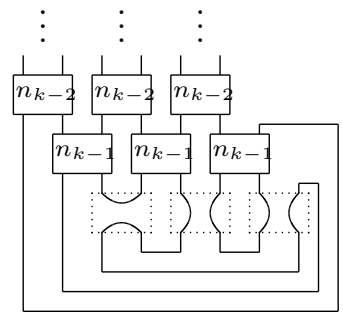

$D_{k}^{(3)}(\infty, 0,0)$

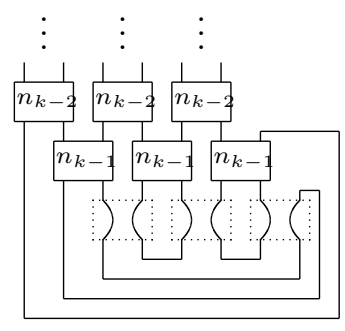

$D_{k}^{(3)}(0,0,0)$

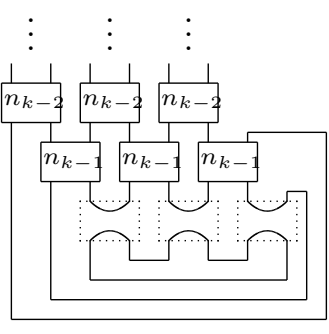

$D_{k}^{(3)}(\infty, \infty, \infty)$

FIGURE 11

For any nonzero integer $n$, let $\mathcal{B}_{n}(t)$ be a Laurent polynomial in $\mathbb{Z}\left[t^{ \pm \frac{1}{2}}\right]$ defined by

$$
\mathcal{B}_{n}(t)= \begin{cases}t^{-1}\left((-t)^{-n}+2\right)\left(\sum_{i=0}^{n-1}(-t)^{-i}\right)^{2} & \text { if } n \geq 1, \\ t\left((-t)^{-n}+2\right)\left(\sum_{i=0}^{-n-1}(-t)^{i}\right)^{2} & \text { if } n \leq-1 .\end{cases}
$$

We note that $\mathcal{B}_{n}(t)=\left((-t)^{-n}+2\right) \mathcal{A}_{n}(t)^{2}$. 


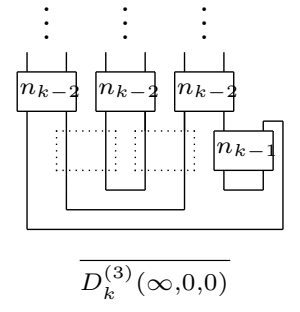

(a)

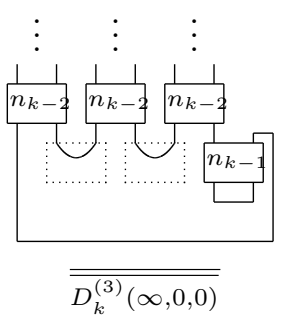

(b)

FIGURE 12

Theorem 5.2. Let $n_{1}, n_{2}, \ldots, n_{r}$ be given nonzero integers. Let $L_{k}^{(3)}$ be 3 periodic link with rational quotient $L_{k}=\vec{C}\left[\left[n_{1}, n_{2}, \ldots, n_{k}\right]\right]$ and let $L_{0}^{(3)}$ the trivial knot. Let $V_{k}(t)$ be the Jones polynomial of $L_{k}^{(3)}$ for each $k=0,1,2, \ldots, r$. Then we have the following recursive formula:

(20) $\quad V_{0}(t)=1$,

(21) $\quad V_{1}(t)=(-t)^{3 n_{1}}\left(\mathcal{B}_{n_{1}}(t)+3(-t)^{-n_{1}}+t^{-1}-1+t\right)$,

$$
V_{k}(t)=(-t)^{3 n_{k}}\left(\mathcal{B}_{n_{k}}(t) V_{k-1}(t)+3 \mathcal{A}_{n_{k}}(t) \lambda_{k}(t)+V_{k-2}(t)\right) \text {, }
$$

where

$$
\begin{aligned}
& \lambda_{1}(t)=-t^{-\frac{1}{2}}-t^{\frac{1}{2}}, \\
& \lambda_{k}(t)=t^{2 n_{k-1}}\left(\lambda_{k-1}(t)+\mathcal{A}_{2 n_{k-1}}(t) V_{k-2}(t)\right) .
\end{aligned}
$$

Proof. For each $k=1,2, \ldots, r$, let $D_{k}^{(3)}$ be the canonical 3-periodic diagram of the 3 -periodic link $L_{k}^{(3)}$ with rational quotient $L=\vec{C}\left[\left[n_{1}, n_{2}, \ldots, n_{k}\right]\right]$. Then

$$
V_{k}(t)=\left.(-A)^{-3 w\left(D_{k}^{(3)}\right)}\left\langle D_{k}^{(3)}\right\rangle\right|_{A=t^{-\frac{1}{4}}} .
$$

For each $k=1,2, \ldots, r$, put $f_{k}(A)=(-A)^{-3 w\left(D_{k}^{(3)}\right)}\left\langle D_{k}^{(3)}\right\rangle$. Then $V_{k}(t)=$ $\left.f_{k}(A)\right|_{A=t^{-\frac{1}{4}}}$. We note that $w\left(D_{k}^{(3)}\right)=3 \sum_{i=1}^{k} n_{i}$ and that, by Lemma 3.2 , $\beta_{n_{i}} \delta+3 \alpha_{n_{i}}=A^{-n_{i}}\left(\left(-A^{4}\right)^{n_{i}}+2\right)$.

Since $L_{0}^{(3)}$ is the trivial knot, $V_{0}(t)=1$. If $n_{1} \geq 1$, then

$$
\begin{aligned}
f_{1}(A)= & (-A)^{-9 n_{1}}\left(\beta_{n_{1}}^{2}\left(3 \alpha_{n_{1}}+\beta_{n_{1}} \delta\right)+\alpha_{n_{1}}^{2} \delta\left(3 \beta_{n_{1}}+\alpha_{n_{1}} \delta\right)\right) \\
= & (-1)^{n_{1}} A^{-12 n_{1}+4}\left(\sum_{i=0}^{n_{1}-1}\left(-A^{4}\right)^{i}\right)^{2}\left(\left(-A^{4}\right)^{n_{1}}+2\right) \\
& +(-1)^{n_{1}} A^{-12 n_{1}+4}\left(-1-A^{-4}\right)\left(3 \sum_{i=0}^{n_{1}-1}\left(-A^{4}\right)^{i}+\left(-1-A^{-4}\right)\right)
\end{aligned}
$$


and hence

$$
\begin{aligned}
V_{1}(t)= & (-1)^{n_{1}} t^{3 n_{1}-1}\left(\sum_{i=0}^{n_{1}-1}(-t)^{-i}\right)^{2}\left((-t)^{-n_{1}}+2\right) \\
& +(-1)^{n_{1}} t^{3 n_{1}-1}(-1-t)\left(3 \sum_{i=0}^{n_{1}-1}(-t)^{-i}+(-1-t)\right) \\
= & (-t)^{3 n_{1}}\left(\mathcal{B}_{n_{1}}(t)+3(-t)^{-n_{1}}+t^{-1}-1+t\right) .
\end{aligned}
$$

If $n_{1} \leq-1$, then

$$
\begin{aligned}
f_{1}(A)= & (-1)^{n_{1}} A^{-12 n_{1}-4}\left(\sum_{i=0}^{-n_{1}-1}\left(-A^{-4}\right)^{i}\right)^{2}\left(\left(-A^{4}\right)^{n_{1}}+2\right) \\
& +(-1)^{n_{1}} A^{-12 n_{1}+4}\left(-1-A^{-4}\right)\left(3 A^{-4} \sum_{i=0}^{-n_{1}-1}\left(-A^{-4}\right)^{i}+\left(-1-A^{-4}\right)\right)
\end{aligned}
$$

and hence

$$
\begin{aligned}
V_{1}(t)= & (-1)^{n_{1}} t^{3 n_{1}+1}\left(\sum_{i=0}^{-n_{1}-1}(-t)^{i}\right)^{2}\left((-t)^{-n_{1}}+2\right) \\
& +(-1)^{n_{1}} t^{3 n_{1}-1}(-1-t)\left(3 t \sum_{i=0}^{-n_{1}-1}(-t)^{i}+(-1-t)\right) \\
= & (-t)^{3 n_{1}}\left(\mathcal{B}_{n_{1}}(t)+3(-t)^{-n_{1}}+t^{-1}-1+t\right) .
\end{aligned}
$$

Put $\lambda_{1}(t)=\left.\delta\right|_{A=t^{-\frac{1}{4}}}$ and $\lambda_{k}(t)=\left.(-A)^{-9 \sum_{i=1}^{k-1} n_{i}} \gamma_{k}\right|_{A=t^{-\frac{1}{4}}}$ for each $k=$ $2,3, \ldots, r$. Then $\lambda_{1}(t)=-t^{-\frac{1}{2}}-t^{\frac{1}{2}}$ and

$$
\begin{aligned}
\lambda_{k}(t)= & \left.(-A)^{-9 \sum_{i=1}^{k-1} n_{i}}(-A)^{3 n_{k-1}} \alpha_{n_{k-1}}^{2} \gamma_{n_{k-1}}\right|_{A=t^{-\frac{1}{4}}} \\
& +\left.(-A)^{-9 \sum_{i=1}^{k-1} n_{i}}(-A)^{3 n_{k-1}} \beta_{n_{k-1}}\left(\beta_{n_{k-1}} \delta+2 \alpha_{n_{k-1}}\right)\left\langle D_{k-2}^{(3)}\right\rangle\right|_{A=t^{-\frac{1}{4}}} \\
= & \left.A^{-8 n_{k-1}}(-A)^{-9 \sum_{i=1}^{k-2} n_{i}} \gamma_{n_{k-1}}\right|_{A=t^{-\frac{1}{4}}} \\
& +\left.A^{-7 n_{k-1}}\left(\left(-A^{4}\right)^{n_{k-1}}+1\right) \beta_{n_{k-1}}(-A)^{-9 \sum_{i=1}^{k-2} n_{i}}\left\langle D_{k-2}^{(3)}\right\rangle\right|_{A=t^{-\frac{1}{4}}} \\
= & t^{2 n_{k-1}} \lambda_{k-1}(t)+t^{2 n_{k-1}}\left((-t)^{-n_{k-1}}+1\right) \mathcal{A}_{n_{k-1}}(t) V_{k-2}(t) \\
= & t^{2 n_{k-1}} \lambda_{k-1}(t)+t^{2 n_{k-1}} \mathcal{A}_{2 n_{k-1}}(t) V_{k-2}(t) .
\end{aligned}
$$


For $k \geq 2$, by Lemma 5.1, we obtain

$$
\begin{aligned}
f_{k}(A)= & (-A)^{-9 \sum_{i=1}^{k} n_{i}}\left(\beta_{n_{k}}^{3} \delta+3 \alpha_{n_{k}} \beta_{n_{k}}^{2}\right)\left\langle D_{k-1}^{(3)}\right\rangle \\
& +(-A)^{-9 \sum_{i=1}^{k} n_{i}} 3 \alpha_{n_{k}}^{2} \beta_{n_{k}} \gamma_{k}+(-A)^{-9 \sum_{i=1}^{k} n_{i}}(-A)^{9 n_{k-1}} \alpha_{n_{k}}^{3}\left\langle D_{k-2}^{(3)}\right\rangle \\
= & (-A)^{-9 n_{k}}\left(\beta_{n_{k}}^{3} \delta+3 \alpha_{n_{k}} \beta_{n_{k}}^{2}\right) f_{k-1}(A) \\
& +(-A)^{-9 \sum_{i=1}^{k} n_{i}} 3 \alpha_{n_{k}}^{2} \beta_{n_{k}} \gamma_{k}+(-A)^{-9 n_{k}} \alpha_{n_{k}}^{3} f_{k-2}(A) .
\end{aligned}
$$

Note that $\left.(-A)^{-9 n_{k}} \alpha_{n_{k}}^{3}\right|_{A=t^{-\frac{1}{4}}}=(-1)^{n_{k}} t^{3 n_{k}}$ and $\left.(-A)^{-9 n_{k}} \alpha_{n_{k}}^{2} \beta_{n_{k}}\right|_{A=t^{-\frac{1}{4}}}=$ $(-1)^{n_{k}} t^{3 n_{k}} \mathcal{A}_{n_{k}}(t)$. If $n_{k} \geq 1$, then by Lemma 3.2 we have

$$
(-A)^{-9 n_{k}}\left(\beta_{n_{k}}^{3} \delta+3 \alpha_{n_{k}} \beta_{n_{k}}^{2}\right)=(-1)^{n_{k}} A^{-12 n_{k}+4}\left(\left(-A^{4}\right)^{n_{k}}+2\right)\left(\sum_{i=0}^{n_{k}-1}\left(-A^{4}\right)^{i}\right)^{2}
$$

and hence

$$
\begin{aligned}
& \left.(-A)^{-9 n_{k}}\left(\beta_{n_{k}}^{3} \delta+3 \alpha_{n_{k}} \beta_{n_{k}}^{2}\right)\right|_{A=t^{-\frac{1}{4}}} \\
= & (-1)^{n_{k}} t^{3 n_{k}-1}\left((-t)^{-n_{k}}+2\right)\left(\sum_{i=0}^{n_{k}-1}(-t)^{-i}\right)^{2} \\
= & (-t)^{3 n_{k}} \mathcal{B}_{n_{k}}(t) .
\end{aligned}
$$

If $n_{k} \leq-1$, then by Lemma 3.2 we also have

$$
(-A)^{-9 n_{k}}\left(\beta_{n_{k}}^{3} \delta+3 \alpha_{n_{k}} \beta_{n_{k}}^{2}\right)=(-1)^{n_{k}} A^{-12 n_{k}-4}\left(\left(-A^{4}\right)^{n_{k}}+2\right)\left(\sum_{i=0}^{-n_{k}-1}\left(-A^{-4}\right)^{i}\right)^{2}
$$

and hence

$$
\begin{aligned}
& \left.(-A)^{-9 n_{k}}\left(\beta_{n_{k}}^{3} \delta+3 \alpha_{n_{k}} \beta_{n_{k}}^{2}\right)\right|_{A=t^{-\frac{1}{4}}} \\
= & (-1)^{n_{k}} t^{3 n_{k}+1}\left((-t)^{-n_{k}}+2\right)\left(\sum_{i=0}^{-n_{k}-1}(-t)^{i}\right)^{2} \\
= & (-t)^{3 n_{k}} \mathcal{B}_{n_{k}}(t) .
\end{aligned}
$$

Therefore we have

$$
\begin{aligned}
V_{k}(t)= & \left.f_{k}(A)\right|_{A=t^{-\frac{1}{4}}} \\
= & \left.(-A)^{-9 n_{k}}\left(\beta_{n_{k}}^{3} \delta+3 \alpha_{n_{k}} \beta_{n_{k}}^{2}\right) f_{k-1}(A)\right|_{A=t^{-\frac{1}{4}}} \\
& +\left.(-A)^{-9 \sum_{i=1}^{k} n_{i}} 3 \alpha_{n_{k}}^{2} \beta_{n_{k}} \gamma_{k}\right|_{A=t^{-\frac{1}{4}}}+\left.(-A)^{-9 n_{k}} \alpha_{n_{k}}^{3} f_{k-2}(A)\right|_{A=t^{-\frac{1}{4}}} \\
= & (-t)^{3 n_{k}} \mathcal{B}_{n_{k}}(t) V_{k-1}(t)+3(-t)^{3 n_{k}} \mathcal{A}_{n_{k}}(t) \lambda_{k}(t)+(-t)^{3 n_{k}} V_{k-2}(t) .
\end{aligned}
$$

This completes the proof. 
Example 5.3. Let $L$ be the 3-periodic knot with rational quotient $\vec{C}[[1,-1,1]]$. Then $L$ is the knot $9_{40}$ in Rolfsen's table [20]. Let $n_{1}=1, n_{2}=-1$ and $n_{3}=1$. Then we have that $\mathcal{A}_{1}(t)=t^{-\frac{1}{2}}, \mathcal{A}_{-1}(t)=t^{\frac{1}{2}}, \mathcal{A}_{2}(t)=t^{-\frac{1}{2}}-t^{-\frac{3}{2}}$, $\mathcal{A}_{-2}(t)=t^{\frac{1}{2}}-t^{\frac{3}{2}}, \mathcal{B}_{1}(t)=2 t^{-1}-t^{-2}$ and $\mathcal{B}_{-1}(t)=2 t-t^{2}$. From Theorem 5.2, we get that $V_{0}(t)=1, V_{1}(t)=-t^{4}+t^{3}+t, \lambda_{2}=-t^{\frac{5}{2}}-t^{\frac{1}{2}}$, $V_{2}(t)=-t^{3}+3 t^{2}-2 t+4-2 t^{-1}+3 t^{-2}-t^{-3}, \lambda_{3}=t^{\frac{7}{2}}-2 t^{\frac{5}{2}}+t^{\frac{3}{2}}-2 t^{\frac{1}{2}}+t^{-\frac{1}{2}}-t^{-\frac{3}{2}}$ and $V_{3}(t)=t^{7}-4 t^{6}+8 t^{5}-11 t^{4}+13 t^{3}-13 t^{2}+11 t-8+5 t^{-1}-t^{-2}$. Hence the Jones polynomial of $L$ is

$$
V_{L}(t)=t^{7}-4 t^{6}+8 t^{5}-11 t^{4}+13 t^{3}-13 t^{2}+11 t-8+5 t^{-1}-t^{-2} .
$$

Let $n$ be any nonzero integer. From (19), we note that

(25) $\quad \max \operatorname{deg} \mathcal{B}_{n}(t)=-1+\frac{3}{2}(|n|-n), \quad \min \operatorname{deg} \mathcal{B}_{n}(t)=1-\frac{3}{2}(|n|+n)$.

From (10), we also note that

(26) $\max \operatorname{deg} \mathcal{A}_{n}(t)=-\frac{1}{2}+\frac{1}{2}(|n|-n), \quad \min \operatorname{deg} \mathcal{A}_{n}(t)=\frac{1}{2}-\frac{1}{2}(|n|+n)$.

Lemma 5.4. For given nonzero integers $n_{1}, n_{2}, \ldots, n_{r}$, let $V_{k}(t)$ be the Jones polynomial of the 3 -periodic link $L_{k}^{(3)}$ with rational quotient $L_{k}=\vec{C}\left[\left[n_{1}, n_{2}, \ldots\right.\right.$, $\left.\left.n_{k}\right]\right]$. Suppose that $n_{i} \neq 1$ for all $i=1,2, \ldots, r$. In the recursive formula in Theorem 5.2, we have the following properties for each $k=2,3, \ldots, r$ :

(1) If $n_{k}>1$ and $n_{k-1} \leq-1$, then $\max \operatorname{deg} \mathcal{B}_{n_{k}}(t) V_{k-1}(t)<\max \operatorname{deg} V_{k-2}(t)$ and $\max \operatorname{deg} \mathcal{A}_{n_{k}}(t) \lambda_{k}(t)<\max \operatorname{deg} V_{k-2}(t)$.

(2) If $n_{k} \leq-1$ or $n_{k-1}>1$, then $\max \operatorname{deg} V_{k-2}(t)<\max \operatorname{deg} \mathcal{B}_{n_{k}}(t) V_{k-1}(t)$ and max $\operatorname{deg} \mathcal{A}_{n_{k}}(t) \lambda_{k}(t)<\max \operatorname{deg} \mathcal{B}_{n_{k}}(t) V_{k-1}(t)$.

Proof. We will use induction on $k$. If $n_{1}>1$, then $\max \operatorname{deg} \mathcal{B}_{n_{1}}(t)=-1$. From (21), we have max $\operatorname{deg} V_{1}(t)=3 n_{1}+1$. If $n_{1} \leq-1$, then $\max \operatorname{deg} \mathcal{B}_{n_{1}}(t)=$ $-1-3 n_{1}$. From (21), we get $\max \operatorname{deg} V_{1}(t)=-1$. Therefore we have

$$
\max \operatorname{deg} V_{1}(t)=\frac{3}{2}\left(\left|n_{1}\right|+n_{1}\right)+\epsilon_{1} .
$$

From (20) and (24), we note that max $\operatorname{deg} \lambda_{2}(t)=\left(\left|n_{1}\right|+n_{1}\right)+\frac{1}{2} \epsilon_{1}$ and $\max \operatorname{deg} V_{0}(t)=0$. If $n_{2}>1$ and $n_{1} \leq-1$, then $\max \operatorname{deg} \mathcal{B}_{n_{2}}(t) V_{1}(t)=-2$ and $\max \operatorname{deg} \mathcal{A}_{n_{2}}(t) \lambda_{2}(t)=-1$. Hence we have that if $n_{2}>1$ and $n_{1} \leq-1$, then

$$
\max \operatorname{deg} \mathcal{B}_{n_{2}}(t) V_{1}(t)<\max \operatorname{deg} V_{0}(t)
$$

and

If $n_{2} \leq-1$, then

$$
\max \operatorname{deg} \mathcal{A}_{n_{2}}(t) \lambda_{2}(t)<\max \operatorname{deg} V_{0}(t)
$$

$$
\max \operatorname{deg} \mathcal{B}_{n_{2}}(t) V_{1}(t)=-1-3 n_{2}+\frac{3}{2}\left(\left|n_{1}\right|+n_{1}\right)+\epsilon_{1}
$$

and

$$
\max \operatorname{deg} \mathcal{A}_{n_{2}}(t) \lambda_{2}(t)=-\frac{1}{2}-n_{2}+\left(\left|n_{1}\right|+n_{1}\right)+\frac{1}{2} \epsilon_{1} .
$$


If $n_{1}>1$, then

$$
\max \operatorname{deg} \mathcal{B}_{n_{2}}(t) V_{1}(t)=\frac{3}{2}\left(\left|n_{2}\right|-n_{2}\right)+3 n_{1}
$$

and

$$
\max \operatorname{deg} \mathcal{A}_{n_{2}}(t) \lambda_{2}(t)=\frac{1}{2}\left(\left|n_{2}\right|-n_{2}\right)+2 n_{1} .
$$

Hence we have that if $n_{2} \leq-1$ or $n_{1}>1$, then

$$
\max \operatorname{deg} V_{0}(t)<\max \operatorname{deg} \mathcal{B}_{n_{2}}(t) V_{1}(t)
$$

and

$$
\max \operatorname{deg} \mathcal{A}_{n_{2}}(t) \lambda_{2}(t)<\max \operatorname{deg} \mathcal{B}_{n_{2}}(t) V_{1}(t) .
$$

Now we assume that the statements hold for $\leq k$. From now on we will prove that the statements hold for $k+1$.

Case (i) : Suppose that $n_{k+1}>1$ and $n_{k} \leq-1$. By the induction hypothesis and (22), we have $\max \operatorname{deg} V_{k}(t)=3 n_{k}+\max \operatorname{deg} \mathcal{B}_{n_{k}}(t) V_{k-1}(t)$. Hence we have

$$
\begin{aligned}
\max \operatorname{deg} \mathcal{B}_{n_{k+1}}(t) V_{k}(t) & =-1+\max \operatorname{deg} V_{k}(t) \\
& =-1+3 n_{k}+\max \operatorname{deg} \mathcal{B}_{n_{k}}(t) V_{k-1}(t) \\
& =-1+3 n_{k}+\left(-1-3 n_{k}\right)+\max \operatorname{deg} V_{k-1}(t) \\
& =-2+\max \operatorname{deg} V_{k-1}(t) \\
& <\max \operatorname{deg} V_{k-1}(t) .
\end{aligned}
$$

From (24), it is true that either $\max \operatorname{deg} \lambda_{k+1}(t) \leq 2 n_{k}+\max \operatorname{deg} \lambda_{k}(t)$ or $\max \operatorname{deg} \lambda_{k+1}(t) \leq 2 n_{k}+\max \operatorname{deg} \mathcal{A}_{2 n_{k}}(t) V_{k-1}(t)$. If $\max \operatorname{deg} \lambda_{k+1}(t) \leq 2 n_{k}+$ $\max \operatorname{deg} \lambda_{k}(t)$, then, by the induction hypothesis, we get

$$
\begin{aligned}
\max \operatorname{deg} \lambda_{k+1}(t) & \leq 2 n_{k}+\max \operatorname{deg} \lambda_{k}(t) \\
& <2 n_{k}-\max \operatorname{deg} \mathcal{A}_{n_{k}}(t)+\max \operatorname{deg} \mathcal{B}_{n_{k}}(t) V_{k-1}(t) \\
& =-\frac{1}{2}+\max \operatorname{deg} V_{k-1}(t)
\end{aligned}
$$

If $\max \operatorname{deg} \lambda_{k+1}(t) \leq 2 n_{k}+\max \operatorname{deg} \mathcal{A}_{2 n_{k}}(t) V_{k-1}(t)$, then we have

$$
\max \operatorname{deg} \lambda_{k+1}(t) \leq-\frac{1}{2}+\max \operatorname{deg} V_{k-1}(t) .
$$

Hence we know

$$
\begin{aligned}
\max \operatorname{deg} \mathcal{A}_{n_{k+1}}(t) \lambda_{k+1}(t) & =-\frac{1}{2}+\max \operatorname{deg} \lambda_{k+1}(t) \\
& \leq-1+\max \operatorname{deg} V_{k-1}(t) \\
& <\max \operatorname{deg} V_{k-1}(t) .
\end{aligned}
$$

By (27) and (28), it follows that the statement (1) holds. 
Case (ii) : Suppose that $n_{k+1} \leq-1$ or $n_{k}>1$. By the induction hypothesis and (22), we have that

$$
\begin{aligned}
\max \operatorname{deg} V_{k}(t) & \geq 3 n_{k}+\max \operatorname{deg} \mathcal{B}_{n_{k}}(t)+\max \operatorname{deg} V_{k-1}(t) \\
& =-1+\frac{3}{2}\left(\left|n_{k}\right|+n_{k}\right)+\max \operatorname{deg} V_{k-1}(t)
\end{aligned}
$$

and

$$
\begin{aligned}
\max \operatorname{deg} \lambda_{k}(t) & \leq \max \operatorname{deg} V_{k}(t)-3 n_{k}-\max \operatorname{deg} \mathcal{A}_{n_{k}}(t) \\
& =\max \operatorname{deg} V_{k}(t)-3 n_{k}+\frac{1}{2}-\frac{1}{2}\left(\left|n_{k}\right|-n_{k}\right)
\end{aligned}
$$

From (29), we get

$$
\begin{aligned}
& \max \operatorname{deg} \mathcal{B}_{n_{k+1}}(t) V_{k}(t) \\
= & -1+\frac{3}{2}\left(\left|n_{k+1}\right|-n_{k+1}\right)+\max \operatorname{deg} V_{k}(t) \\
\geq & -1+\frac{3}{2}\left(\left|n_{k+1}\right|-n_{k+1}\right)-1+\frac{3}{2}\left(\left|n_{k}\right|+n_{k}\right)+\max \operatorname{deg} V_{k-1}(t) \\
= & -2+\frac{3}{2}\left(\left|n_{k+1}\right|-n_{k+1}\right)+\frac{3}{2}\left(\left|n_{k}\right|+n_{k}\right)+\max \operatorname{deg} V_{k-1}(t) \\
> & \max \operatorname{deg} V_{k-1}(t)
\end{aligned}
$$

From (24), we know that either

$$
\max \operatorname{deg} \lambda_{k+1}(t) \leq 2 n_{k}+\max \operatorname{deg} \lambda_{k}(t)
$$

or

$$
\max \operatorname{deg} \lambda_{k+1}(t) \leq 2 n_{k}+\max \operatorname{deg} \mathcal{A}_{2 n_{k}}(t)+\max \operatorname{deg} V_{k-1}(t) .
$$

If $\max \operatorname{deg} \lambda_{k+1}(t) \leq 2 n_{k}+\max \operatorname{deg} \lambda_{k}(t)$, then from (30), we calculate

$$
\begin{aligned}
\max \operatorname{deg} \lambda_{k+1}(t) \leq & 2 n_{k}+\max \operatorname{deg} \lambda_{k}(t) \\
\leq & 2 n_{k}+\max \operatorname{deg} V_{k}(t)-3 n_{k}+\frac{1}{2}-\frac{1}{2}\left(\left|n_{k}\right|-n_{k}\right) \\
= & \max \operatorname{deg} V_{k}(t)+\frac{1}{2}-\frac{1}{2}\left(\left|n_{k}\right|+n_{k}\right) \\
= & \max \operatorname{deg} \mathcal{B}_{n_{k+1}}(t) V_{k}(t)-\max \operatorname{deg} \mathcal{A}_{n_{k+1}}(t) \\
& +\left(1-\frac{1}{2}\left(\left|n_{k}\right|+n_{k}\right)-\left(\left|n_{k+1}\right|-n_{k+1}\right)\right)
\end{aligned}
$$


If $\max \operatorname{deg} \lambda_{k+1}(t) \leq 2 n_{k}+\max \operatorname{deg} \mathcal{A}_{2 n_{k}}(t)+\max \operatorname{deg} V_{k-1}(t)$, then from $(29)$, we have

$$
\begin{aligned}
\max \operatorname{deg} \lambda_{k+1}(t) \leq & 2 n_{k}+\max \operatorname{deg} \mathcal{A}_{2 n_{k}}(t)+\max \operatorname{deg} V_{k-1}(t) \\
\leq & 2 n_{k}-\frac{1}{2}+\left(\left|n_{k}\right|-n_{k}\right)+1-\frac{3}{2}\left(\left|n_{k}\right|+n_{k}\right)+\max \operatorname{deg} V_{k}(t) \\
= & \frac{1}{2}-\frac{1}{2}\left(\left|n_{k}\right|+n_{k}\right)+\max \operatorname{deg} V_{k}(t) \\
= & \max \operatorname{deg} \mathcal{B}_{n_{k+1}}(t) V_{k}(t)-\max \operatorname{deg} \mathcal{A}_{n_{k+1}}(t) \\
& +\left(1-\frac{1}{2}\left(\left|n_{k}\right|+n_{k}\right)-\left(\left|n_{k+1}\right|-n_{k+1}\right)\right)
\end{aligned}
$$

Since $n_{k+1} \leq-1$ or $n_{k}>1$, we get that $1-\frac{1}{2}\left(\left|n_{k}\right|+n_{k}\right)-\left(\left|n_{k+1}\right|-n_{k+1}\right)<0$ and hence

$$
\max \operatorname{deg} \mathcal{A}_{n_{k+1}}(t) \lambda_{k+1}(t)<\max \operatorname{deg} \mathcal{B}_{n_{k+1}}(t) V_{k}(t) .
$$

By (31) and (32), it follows that the statement (2) holds. This completes the proof.

Lemma 5.5. For given nonzero integers $n_{1}, n_{2}, \ldots, n_{r}$, let $V_{k}(t)$ be the Jones polynomial of the 3 -periodic link $L_{k}^{(3)}$ with rational quotient $L_{k}=\vec{C}\left[\left[n_{1}, n_{2}, \ldots\right.\right.$, $\left.n_{k}\right]$ ]. Suppose that $n_{i} \neq-1$ for all $i=1,2, \ldots, r$. In the recursive formula in Theorem 5.2, we have the following properties for each $k=2,3, \ldots, r$ :

(1) If $n_{k}<-1$ and $n_{k-1} \geq 1$, then $\min \operatorname{deg} \mathcal{B}_{n_{k}}(t) V_{k-1}(t)>\min \operatorname{deg} V_{k-2}(t)$ and $\min \operatorname{deg} \mathcal{A}_{n_{k}}(t) \lambda_{k}(t)>\min \operatorname{deg} V_{k-2}(t)$.

(2) If $n_{k} \geq 1$ or $n_{k-1}<-1$, then $\min \operatorname{deg} V_{k-2}(t)>\min \operatorname{deg} \mathcal{B}_{n_{k}}(t) V_{k-1}(t)$ and $\min \operatorname{deg} \mathcal{A}_{n_{k}}(t) \lambda_{k}(t)>\min \operatorname{deg} \mathcal{B}_{n_{k}}(t) V_{k-1}(t)$.

Proof. Let $m_{i}=-n_{i}$ for all $i=1,2, \ldots, r$ and let $\tilde{V}_{k}(t)$ be the Jones polynomial of the 3 -periodic link $\tilde{L}_{k}^{(3)}$ with rational quotient $\tilde{L}_{k}=\vec{C}\left[\left[m_{1}, m_{2}, \ldots, m_{k}\right]\right]$. Let $\tilde{\lambda}_{k}(t)$ be the Laurent polynomial recursively defined by

$$
\tilde{\lambda}_{1}(t)=-t^{-\frac{1}{2}}-t^{\frac{1}{2}}, \tilde{\lambda}_{k}(t)=t^{2 m_{k-1}}\left(\tilde{\lambda}_{k-1}(t)+\mathcal{A}_{2 m_{k-1}}(t) \tilde{V}_{k-2}(t)\right) .
$$

By (25) and (26),

$\max \operatorname{deg} \mathcal{B}_{m_{k}}(t)=-\min \operatorname{deg} \mathcal{B}_{n_{k}}(t), \max \operatorname{deg} \mathcal{A}_{m_{k}}(t)=-\min \operatorname{deg} \mathcal{A}_{n_{k}}(t)$.

We observe that $\tilde{L}_{k}^{(3)}$ is the mirror image of $L_{k}^{(3)}$ and hence $\tilde{V}_{k}(t)=V_{k}\left(t^{-1}\right)$. Therefore

$$
\max \operatorname{deg} \tilde{V}_{k}(t)=-\min \operatorname{deg} V_{k}(t), \max \operatorname{deg} \tilde{\lambda}_{k}(t)=-\min \operatorname{deg} \lambda_{k}(t) .
$$

By Lemma 5.4,

(1) if $m_{k}>1$ and $m_{k-1} \leq-1$, then $\max \operatorname{deg} \mathcal{B}_{m_{k}}(t) \tilde{V}_{k-1}(t)<\max \operatorname{deg} \tilde{V}_{k-2}(t)$ and $\max \operatorname{deg} \mathcal{A}_{m_{k}}(t) \tilde{\lambda}_{k}(t)<\max \operatorname{deg} \tilde{V}_{k-2}(t)$,

(2) if $m_{k} \leq-1$ or $m_{k-1}>1$, then max $\operatorname{deg} \tilde{V}_{k-2}(t)<\max \operatorname{deg} \mathcal{B}_{m_{k}}(t) \tilde{V}_{k-1}(t)$ and $\max \operatorname{deg} \mathcal{A}_{m_{k}}(t) \tilde{\lambda}_{k}(t)<\max \operatorname{deg} \mathcal{B}_{m_{k}}(t) \tilde{V}_{k-1}(t)$. 
This completes the proof.

Theorem 5.6. For given nonzero integers $n_{1}, n_{2}, \ldots, n_{r}$, let $V_{k}(t)$ be the Jones polynomial of the 3 -periodic link $L_{k}^{(3)}$ with rational quotient $L_{k}=\vec{C}\left[\left[n_{1}, n_{2}, \ldots\right.\right.$, $\left.n_{k}\right]$ ]. Suppose that $n_{i} \neq \pm 1$ for all $i=1,2, \ldots, r$. Then

$$
\max \operatorname{deg} V_{k}(t)=(1-k)+\frac{3}{2} \sum_{i=1}^{k}\left(n_{i}+\left|n_{i}\right|\right)+\epsilon_{1}+\frac{1}{2} \sum_{j=1}^{k-1}\left(1-\epsilon_{j}\right)\left(1+\epsilon_{j+1}\right)
$$

and

$$
\min \operatorname{deg} V_{k}(t)=(k-1)+\frac{3}{2} \sum_{i=1}^{k}\left(n_{i}-\left|n_{i}\right|\right)+\epsilon_{1}-\frac{1}{2} \sum_{j=1}^{k-1}\left(1+\epsilon_{j}\right)\left(1-\epsilon_{j+1}\right) .
$$

Proof. In the proof of Lemma 5.4, we have

$$
\max \operatorname{deg} V_{1}(t)=\frac{3}{2}\left(\left|n_{1}\right|+n_{1}\right)+\epsilon_{1} .
$$

If $n_{2}>1$ and $n_{1}<-1$, then by (1) in Lemma 5.4 we obtain

$$
\max \operatorname{deg} V_{2}(t)=3 n_{2}+\max \operatorname{deg} V_{0}(t)=\frac{3}{2}\left(n_{2}+\left|n_{2}\right|\right) .
$$

If $n_{2}<-1$ or $n_{1}>1$, then by (2) in Lemma 5.4 we get

$$
\begin{aligned}
\max \operatorname{deg} V_{2}(t) & =3 n_{2}+\max \operatorname{deg} \mathcal{B}_{n_{2}}(t) V_{1}(t) \\
& =3 n_{2}+\left(-1+\frac{3}{2}\left(\left|n_{2}\right|-n_{2}\right)+\frac{3}{2}\left(\left|n_{1}\right|+n_{1}\right)+\epsilon_{1}\right) \\
& =-1+\frac{3}{2}\left(\left|n_{2}\right|+n_{2}\right)+\frac{3}{2}\left(\left|n_{1}\right|+n_{1}\right)+\epsilon_{1} .
\end{aligned}
$$

Therefore we have

$$
\max \operatorname{deg} V_{2}(t)=-1+\frac{3}{2} \sum_{i=1}^{2}\left(n_{i}+\left|n_{i}\right|\right)+\epsilon_{1}+\frac{1}{2}\left(1-\epsilon_{1}\right)\left(1+\epsilon_{2}\right) .
$$

If $n_{k+1}>1$ and $n_{k}<-1$, then by (1) in Lemma 5.4 we get

$$
\begin{aligned}
\max \operatorname{deg} V_{k+1}(t)= & 3 n_{k+1}+\max \operatorname{deg} V_{k-1}(t) \\
= & 3 n_{k+1}+(2-k)+\frac{3}{2} \sum_{i=1}^{k-1}\left(n_{i}+\left|n_{i}\right|\right)+\epsilon_{1} \\
& +\frac{1}{2} \sum_{j=1}^{k-2}\left(1-\epsilon_{j}\right)\left(1+\epsilon_{j+1}\right) .
\end{aligned}
$$


If $n_{k+1}<-1$ or $n_{k}>1$, then by (2) in Lemma 5.4 we calculate

$$
\begin{aligned}
\max \operatorname{deg} V_{k+1}(t)= & 3 n_{k+1}+\max \operatorname{deg} \mathcal{B}_{n_{k+1}}(t) V_{k}(t) \\
= & -1+\frac{3}{2}\left(\left|n_{k+1}\right|+n_{k+1}\right)+(1-k) \\
& +\frac{3}{2} \sum_{i=1}^{k}\left(n_{i}+\left|n_{i}\right|\right)+\epsilon_{1}+\frac{1}{2} \sum_{j=1}^{k-1}\left(1-\epsilon_{j}\right)\left(1+\epsilon_{j+1}\right) .
\end{aligned}
$$

Therefore we have

$$
\max \operatorname{deg} V_{k+1}(t)=-k+\frac{3}{2} \sum_{i=1}^{k+1}\left(n_{i}+\left|n_{i}\right|\right)+\epsilon_{1}+\frac{1}{2} \sum_{j=1}^{k}\left(1-\epsilon_{j}\right)\left(1+\epsilon_{j+1}\right) .
$$

By a similar argument, we also have

$$
\min \operatorname{deg} V_{k+1}(t)=k+\frac{3}{2} \sum_{i=1}^{k+1}\left(n_{i}-\left|n_{i}\right|\right)+\epsilon_{1}-\frac{1}{2} \sum_{j=1}^{k}\left(1+\epsilon_{j}\right)\left(1-\epsilon_{j+1}\right) .
$$

This completes the proof.

Theorem 5.7. For given nonzero integers $n_{1}, n_{2}, \ldots, n_{r}$, let $L^{(3)}$ be 3 -periodic link with rational quotient $L=\vec{C}\left[\left[n_{1}, n_{2}, \ldots, n_{r}\right]\right]$. If $\left|n_{i}\right| \geq 2$ for all $i=$ $1,2, \ldots, r$, then the span of the Jones polynomial $V_{L^{(3)}}(t)$ of $L^{(3)}$ is given by

$$
\operatorname{span} V_{L^{(3)}}(t)=3 \sum_{i=1}^{r}\left|n_{i}\right|-2 \kappa\left(n_{i} ; r\right) \text {. }
$$

Proof. From Theorem 5.6, we have

$\operatorname{span} V_{L^{(3)}}(t)=\max \operatorname{deg} V_{r}(t)-\min \operatorname{deg} V_{r}(t)$

$$
\begin{aligned}
= & (1-r)+\frac{3}{2} \sum_{i=1}^{r}\left(n_{i}+\left|n_{i}\right|\right)+\epsilon_{1}+\frac{1}{2} \sum_{j=1}^{r-1}\left(1-\epsilon_{j}\right)\left(1+\epsilon_{j+1}\right) \\
& -(r-1)-\frac{3}{2} \sum_{i=1}^{r}\left(n_{i}-\left|n_{i}\right|\right)-\epsilon_{1}+\frac{1}{2} \sum_{j=1}^{r-1}\left(1+\epsilon_{j}\right)\left(1-\epsilon_{j+1}\right) \\
= & 3 \sum_{i=1}^{r}\left|n_{i}\right|-2 \kappa\left(n_{i} ; r\right)
\end{aligned}
$$

because

$$
\kappa\left(n_{i} ; r\right)=r-1-\frac{1}{4} \sum_{j=1}^{r-1}\left[\left(1-\epsilon_{j}\right)\left(1+\epsilon_{j+1}\right)+\left(1+\epsilon_{j}\right)\left(1-\epsilon_{j+1}\right)\right]
$$

as we have seen in the proof of Theorem 4.5. This completes the proof. 
Acknowledgments. The authors would like to thank the referee for many valuable comments.

\section{References}

[1] J. H. Conway, An enumeration of knots and links, and some of their algebraic properties, 1970 Computational Problems in Abstract Algebra (Proc. Conf., Oxford, 1967) pp. 329358 Pergamon, Oxford.

[2] H. M. Hilden, M. T. Lozano, and J. M. Montesinos-Amilibia, On the character variety of periodic knots and links, Math. Proc. Cambridge Philos. Soc. 129 (2000), no. 3, $477-490$.

[3] H. J. Jang, S. Y. Lee, and M. Seo, Casson knot invariants of periodic knots with rational quotients, J. Knot Theory Ramifications 16 (2007), no. 4, 439-460.

[4] V. F. R. Jones, A polynomial invariant for knots via von Neumann algebras, Bull. Amer. Math. Soc. (N.S.) 12 (1985), no. 1, 103-111.

[5] T. Kanenobu, Examples on polynomial invariants of knots and links. II, Osaka J. Math. 26 (1989), no. 3, 465-482.

[6] - Jones and Q polynomials for 2-bridge knots and links, Proc. Amer. Math. Soc. 110 (1990), no. 3, 835-841.

[7] T. Kanenobu and Y. Miyazawa, 2-bridge link projections, Kobe J. Math. 9 (1992), no. $2,171-182$.

[8] L. H. Kauffman, State models and the Jones polynomial, Topology 26 (1987), no. 3, 395-407.

[9] S. Y. Lee, M.-S. Park, and M. Seo, The Seifert matrices of periodic links with rational quotients, Kyungpook Math. J. 47 (2007), no. 2, 295-309.

[10] S. Y. Lee and M. Seo, Recurrence formulas for the Alexander polynomials of 2-bridge links and their covering links, J. Knot Theory Ramifications 15 (2006), no. 2, 179-203.

[11] _ C Casson knot invariants of periodic knot with rational quotients II, J. Knot Theory Ramifications 17 (2008), no. 8, 905-923.

[12] — The genus of periodic links with rational quotients, to appear in Bull. Austral. Math. Soc. (2009).

[13] W. B. R. Lickorish and K. C. Millett, A polynomial invariant of oriented links, Topology 26 (1987), no. 1, 107-141.

[14] B. Lu and J. K. Zhong, The Kauffman Polynomials of 2-bridge Knots, arXiv:math. GT/0606114.

[15] K. Murasugi, Jones polynomials and classical conjectures in knot theory, Topology 26 (1987), no. 2, 187-194.

[16] _ Jones polynomials of periodic links, Pacific J. Math. 131 (1988), no. 2, 319-329.

[17] _ Knot Theory and its Applications, Translated from the 1993 Japanese original by Bohdan Kurpita. Birkh'äuser Boston, Inc., Boston, MA, 1996.

[18] S. Nakabo, Formulas on the HOMFLY and Jones polynomials of 2-bridge knots and links, Kobe J. Math. 17 (2000), no. 2, 131-144

[19] _ Explicit description of the HOMFLY polynomials for 2-bridge knots and links, J. Knot Theory Ramifications 11 (2002), no. 4, 565-574.

[20] D. Rolfsen, Knots and Links, Mathematics Lecture Series, No. 7. Publish or Perish, Inc., Berkeley, Calif., 1976.

[21] H. Schubert, Knoten mit zwei Brücken, Math Z. 65 (1956), 133-170.

[22] A. Stoimenow, Rational knots and a theorem of Kanenobu, Experiment. Math. 9 (2000), no. $3,473-478$.

[23] M. B. Thistlethwaite, Kauffman's polynomial and alternating links, Topology 27 (1988), no. $3,311-318$. 
EunJu LeE

Department of Mathematics

Graduate School of Natural Sciences

Pusan National University

PUSAN 609-735, KoreA

SANG YOUL LEE

Department of Mathematics

Pusan National University

PUSAN 609-735, KoreA

E-mail address: sangyoul@pusan.ac.kr

Myoungsoo SeO

Department of Mathematics

KyUnGPOOK NATIONAL University

DAegu 702-701, Korea

E-mail address: myseo@knu.ac.kr 